Review

\title{
Scoping Review of Intervention Strategies for Improving Coverage and Uptake of Maternal Nutrition Services in Southeast Asia
}

\author{
Kauma Kurian ${ }^{1}{ }^{(D}$, Theophilus Lakiang ${ }^{1}$, Rajesh Kumar Sinha ${ }^{1} \mathbb{D}^{\text {, Nishtha Kathuria }}{ }^{1} \mathbb{D}$, Priya Krishnan $^{1}$, \\ Devika Mehra $^{1}$, Sunil Mehra ${ }^{1}$ and Shantanu Sharma ${ }^{1,2, * \mathbb{D}}$
}

1 MAMTA Health Institute for Mother and Child, Delhi 110048, India; kurian.kauma@gmail.com (K.K.); theo.lakiang@gmail.com (T.L.); cmarajesh@gmail.com (R.K.S.); nishthak@mamtahimc.in (N.K.); priyakrishnan76@gmail.com (P.K.); devika.mehra@mamtahimc.in (D.M.); dr_mehra@mamtahimc.in (S.M.)

2 Department of Clinical Sciences, Lund University, Skåne University Hospital, S-20502 Malmo, Sweden

* Correspondence: shantanusharma145@gmail.com

Citation: Kurian, K.; Lakiang, T.; Sinha, R.K.; Kathuria, N.; Krishnan, P.; Mehra, D.; Mehra, S.; Sharma, S. Scoping Review of Intervention Strategies for Improving Coverage and Uptake of Maternal Nutrition Services in Southeast Asia. Int. J. Environ. Res. Public Health 2021, 18, 13292. https://doi.org/10.3390/ ijerph182413292

Academic Editors: Scott Rozelle and Arpita Basu

Received: 12 October 2021

Accepted: 12 December 2021

Published: 16 December 2021

Publisher's Note: MDPI stays neutra with regard to jurisdictional claims in published maps and institutional affiliations.

Copyright: (c) 2021 by the authors. Licensee MDPI, Basel, Switzerland. This article is an open access article distributed under the terms and conditions of the Creative Commons Attribution (CC BY) license (https:/ / creativecommons.org/licenses/by/ $4.0 /)$.

\begin{abstract}
Maternal undernutrition can lead to protein-energy malnutrition, micronutrient deficiencies, or anemia during pregnancy or after birth. It remains a major problem, despite evidence-based maternal-nutrition interventions happening on ground. We conducted a scoping review to understand different strategies and delivery mechanisms to improve maternal nutrition, as well as how interventions have improved coverage and uptake of services. An electronic search was conducted in PubMed and Google Scholar for published studies reporting on the effectiveness of maternalnutrition interventions in terms of access or coverage, health outcomes, compliance, and barriers to intervention utilization. The search was limited to studies published within ten years before the initial search date, 8 November 2019; later, it was updated to 17 February 2021. Of 31 studies identified following screening and data extraction, 22 studies were included for narrative synthesis. Twelve studies were reported from India and eleven from Bangladesh, three from Nepal, two from both Pakistan and Thailand (Myanmar), and one from Indonesia. Nutrition education and counselling, home visits, directly observed supplement intake, community mobilization, food, and conditional cash transfer by community health workers were found to be effective. There is a need to incorporate diverse strategies, including various health education approaches, supplementation, as well as strengthening of community participation and the response of the health system in order to achieve impactful maternal nutrition programs.
\end{abstract}

Keywords: community mobilization; counselling; coverage; home visits; maternal nutrition

\section{Introduction}

Maternal nutrition refers to increased nutritional demand during adolescence, antenatal, and postnatal periods. A shortfall in nutrition during these periods can lead to protein-energy malnutrition, micronutrient deficiencies, poor weight gain, or anemia [1]. In low- and middle-income countries, 450 million women are estimated to have short stature, 240 million are underweight (Body Mass Index-BMI $<18.5 \mathrm{~kg} / \mathrm{m}^{2}$ ), and 468 million are anemic [2]. This could be attributed, in part, to the poor performance of maternal nutrition programs in low- and middle-income countries. For example, only $31 \%$ of pregnant women in low- and middle-income countries consumed iron-folic acid tablets for $90+$ days during pregnancy. Similarly, 19\% of the population in Asia and 56\% in Africa are food-insecure. Countries in Asia, like India and Bangladesh, and in Africa, like Ethiopia, Burkina Faso, Liberia, and South Sudan, etc., have some of the lowest coverage rates of one or more key interventions and practices addressing maternal and child malnutrition [3].

Maternal undernutrition is remarkably high in countries of South Asia, with Bangladesh reporting chronic energy deficiency among $>30 \%$ of women of child-bearing age. Likewise, 
more than $16 \%$ of pregnant and lactating women are malnourished in Pakistan, $40 \%$ are anemic, and $12-22 \%$ are chronically energy deficient in Indonesia [1,4]. India also faces a high burden of maternal undernutrition, with more than 100 million adult women with a $\mathrm{BMI}<18.5 \mathrm{~kg} / \mathrm{m}^{2}$ [2]. Considering the magnitude of the problem, addressing maternal undernutrition has become a global priority. Sustainable Development Goal 3 has set a target of ending all forms of malnutrition and addressing the nutritional needs of adolescent girls and pregnant and lactating women by 2030 [5]. India has made strenuous efforts in this direction, and a decline in the prevalence of anemia and undernutrition $\left(\mathrm{BMI}<18.5 \mathrm{~kg} / \mathrm{m}^{2}\right)$ among unmarried adolescents was achieved from 2005 to 2015 [6].

There is a need for a multi-pronged approach to address maternal undernutrition, given its multiple basic and underlying risk factors, as defined by the United Nations Children's Fund (UNICEF) [7]. The risk factors associated with maternal undernutrition include low educational status, poverty, age between 15 and 34 years, living in rural areas, history of multiple pregnancies, parasitic intestinal infections, anemia, limited access to clean drinking water, limited access to nutritious and safe diet, poor marital conditions, lack of decision-making autonomy, and substance abuse [8-12]. The World Health Organization's (WHO) latest guidelines on antenatal care (ANC) recommend fourteen evidence-based nutrition interventions towards a healthy pregnancy, including nutrition counselling, iron and folic acid (IFA), and calcium supplementation, etc. Additionally, emerging evidence recommends pregnant women consume balanced energy and protein supplements (BEP), like ready-to-cook or ready-to-eat food. BEP supplementation is a strategic approach to address chronic energy and protein deficiency among undernourished pregnant women [2].

A past review highlighted those interventions emphasizing counselling or delivery of information to women or their family members, such as consumption of iron-folic acid and calcium tablets by pregnant women for improved nutrition outcomes. Use of counselling material based on locally relevant information and engaging influencers, like mothersin-law and husbands, are effective approaches to improve maternal nutrition outcomes. Furthermore, home visits and use of community forums for greater participation enhance access to services for pregnant women. However, digital interventions in silos are not proven to be an effective approach. The studies included in the review were limited and focused on either IFA or calcium supplementation or access to information concerning diet. Additionally, most of the available evidence reported in the review is from India, with limited data from other countries, like Pakistan, Indonesia, or Bangladesh [13].

To deliver evidence-based maternal-nutrition interventions, various implementation strategies have been initiated, yet maternal undernutrition continues to be a burden. Furthermore, it is imperative to understand and address maternal undernutrition due to its transgenerational impact, resulting in low-birth-weight babies, premature births, and chronic anemia [14]. Studies on the efficacy and effectiveness of these intervention strategies have been conducted in the past. However, to the best of our knowledge, there was lacking a review of the effectiveness of delivery mechanism(s) of such evidence-based maternal-nutrition interventions within the Southeast Asia region. Therefore, we conducted a scoping review to understand the various strategies to improve maternal nutrition, effective delivery mechanisms to improve maternal nutrition, and how interventions have improved coverage and uptake of services, as well as to document barriers and possible solutions for making such delivery mechanisms more effective.

\section{Materials and Methods}

This scoping review was developed and reported in accordance with Preferred Reporting Items for Systematic Review and Meta-analysis extension for scoping review (PRISMA-ScR) guidelines [15].

\subsection{Research Question}

The research question for this review was 'What was the effectiveness of the different strategies for delivering facility- or community-based maternal-nutrition interven- 
tions/programs in countries of Southeast Asia?'. Additionally, 'What were the outcomes, methodological approaches, and characteristics of the interventions/programs?'.

\subsection{Information Sources and Search Strategy}

A search strategy was developed by combining both MeSH terms and free-text words related to pregnancy and components of the nutrition program. An electronic search was performed in PubMed, Google Scholar, and the eLENA library, along with snowballing of relevant articles. The initial search was conducted on 08 November 2019; later, it was updated to 17 February 2021. We searched for articles that were published between 7 November 2010 and 7 November 2019 (last 10 years), later updated to 17 February 2021. The search was limited to the last 10+ years because of a lack of resources; furthermore, the authors perceived that interventions earlier than $10+$ years might not be as relevant in the current scenario. The search was limited to studies published in English within 10+ years prior to the search date.

\subsection{Eligibility Criteria}

Peer-reviewed studies reporting strategies intended to improve maternal nutrition and/or increase coverage, uptake, and compliance with maternal nutrition services during pregnancy were included in this study. Studies of all design types, including randomized controlled trials (RCT), quasi-experimental trials, before-after studies, cross-sectional, casecontrol, etc., were included. All reviews, meta-analyses, letters, editorials, commentaries, conference papers, and reports were excluded. Studies reported from the WHO Southeast Asia region and Pakistan were considered for this review. The final search string used for PubMed can be found in the Supplementary Materials (Table S1).

\subsection{Study-Selection Process}

Screening of titles and abstracts was performed independently by two reviewers against the agreed inclusion and exclusion criteria. During each stage of screening, studies were taken forward only when both reviewers reached a mutual agreement for inclusion. A third reviewer arbitrated any disagreements between the reviewers to reach a consensus. Only those studies approved by both authors were included in the review, and the reason for the exclusion of studies was provided only at the full-text screening stage.

\subsection{Data-Extraction Process}

A standardized, pre-tested data-extraction form was developed. Data were extracted by one reviewer and verified by the other two reviewers. Categories under which data were extracted included (a) study characteristics, (b) methodological characteristics, (c) intervention strategies, and (d) targeted outcome of this review.

\subsection{Collating, Summarizing, and Reporting the Results}

A thematic narrative synthesis of included articles summarizing the effectiveness of each intervention strategy was carefully extracted from each included article. Intervention strategies from all the articles addressing each type of maternal-nutrition intervention were listed and coded.

\section{Results}

Search results: A total of 381 papers were identified from PubMed search, and eight from Google Scholar and reference snowballing. Of 381 papers, 315 were screened based on title and abstract (Figure 1). After the titles and abstracts were reviewed, 231 articles were excluded. During full-text screening, of the remaining 84 articles, 53 articles were excluded, leaving 31 articles for data extraction and quality appraisal. Finally, 22 studies were included for narrative synthesis. 


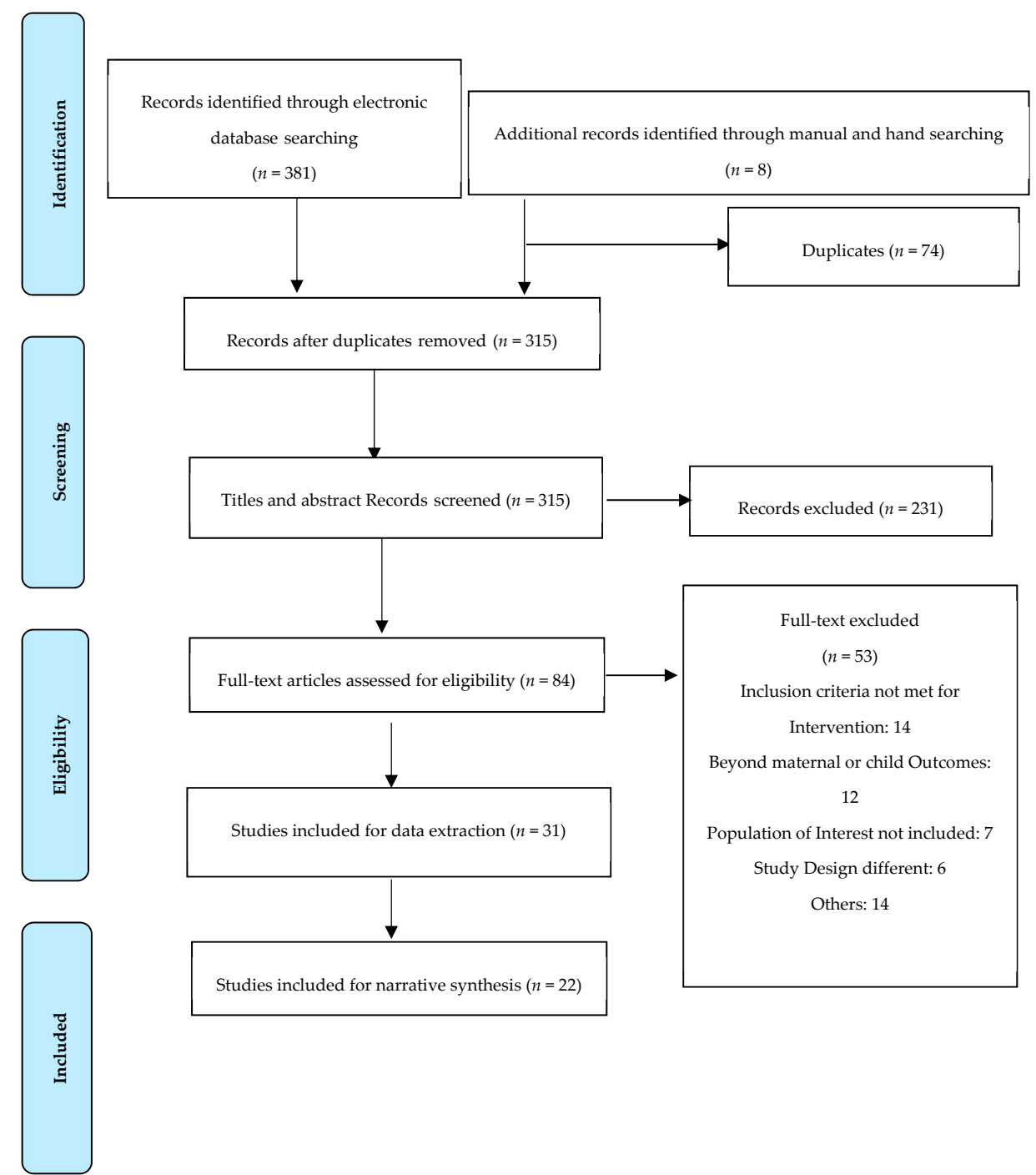

Figure 1. A PRISMA flow chart illustrating search and screening results.

\subsection{Quality Appraisal}

An appraisal was conducted for all papers included after full-text screening. The quality of evidence of each article included in the review was assessed independently by two reviewers using the Joanna Briggs Institute quality-appraisal tool [16]. The overall quality was good, as most of the articles satisfied the criteria in the checklist (Supplementary Materials: Tables S1-S5). There was no disagreement between the two reviewers concerning the inclusion of papers based on the quality checks.

\subsection{Characteristics of Included Articles}

Of all the 31 studies included in the review, only 22 have at least one outcome associated with delivery mechanisms; the study characteristics of all the 31 papers are summarized in Tables 1 and 2 [17-47]. A total of 22 studies were ultimately included in this review for narrative synthesis $[17,18,20-25,27-29,32-34,36,37,39,40,42,43,45,47]$. Of these, twelve studies were RCTs, five were quasi-experimental trials, five were cross-sectional studies, three were mixed-method studies, three were secondary data analyses, one was a cohort study, and two were cross-sectional intervention/comparison studies. The majority (74\%) of the studies was reported from India (12) and Bangladesh (11), three from Nepal, two from Pakistan (two others with India and Nepal), one from Indonesia, and two from Thailand (Myanmar). All identified studies were primarily quantitative, with a few studies reporting qualitative data as well. 
Table 1. Characteristics of the included studies $(n=16)$.

\begin{tabular}{|c|c|c|c|c|c|c|c|c|}
\hline Authors & $\begin{array}{l}\text { Study } \\
\text { Design }\end{array}$ & $\begin{array}{l}\text { Sample } \\
\text { Size }\end{array}$ & Study Population & $\begin{array}{c}\text { Location of the } \\
\text { Study }\end{array}$ & $\begin{array}{l}\text { Intervention/ } \\
\text { Comparison }\end{array}$ & Study Outcomes & $\begin{array}{c}\text { Delivery } \\
\text { Mechanism }\end{array}$ & $\begin{array}{c}\text { Change in } \\
\text { Coverage/Uptake }\end{array}$ \\
\hline $\begin{array}{c}\text { Bhutta, et al. } \\
\text { (2009) [17] }\end{array}$ & Cluster RCT & $\begin{array}{l}\text { I: } 1148 \\
\text { C: } 1230\end{array}$ & Pregnant women & $\begin{array}{l}\text { Pakistan (rural } \\
\text { and urban) }\end{array}$ & $\begin{array}{l}\text { I: Multiple } \\
\text { micronutrients } \\
\text { C: IFA only }\end{array}$ & $\begin{array}{l}\text { Increased maternal monthly weight } \\
\text { gain and reduced LBW prevalence in } \\
\text { the intervention group; no significant } \\
\text { improvement in iron status of } \\
\text { women, but the control group had a } \\
\text { higher prevalence of subclinical } \\
\text { zinc deficiency. }\end{array}$ & $\begin{array}{c}\text { Trained female } \\
\text { CHW } \\
\text { visited fortnightly }\end{array}$ & $\begin{array}{l}\text { No significant } \\
\text { difference in } \\
\text { uptake of ANC } \\
\text { visits or } \\
\text { consumption of } \\
\text { IFA tablets }\end{array}$ \\
\hline $\begin{array}{l}\text { Sunawang, } \\
\text { et al. (2009) } \\
{[18]}\end{array}$ & Cluster RCT & $\begin{array}{l}\text { I: } 432 \\
\text { C: } 411\end{array}$ & Pregnant women & Indonesia & $\begin{array}{l}\text { I: Multiple } \\
\text { micronutrients } \\
\text { C: IFA only }\end{array}$ & $\begin{array}{l}\text { No statistical difference in the birth } \\
\text { parameters of child (birth weight, } \\
\text { length, head circumference), } \\
\text { pregnancy outcome (miscarriage, } \\
\text { stillbirth, or neonatal death), or } \\
\text { maternal hemoglobin or serum levels } \\
\text { of zinc, retinol and ferritin, and } \\
\text { urinary iodine. }\end{array}$ & $\begin{array}{l}\text { Field workers of } \\
\text { the project visited } \\
\text { daily } \\
\text { (except Sunday) }\end{array}$ & $\begin{array}{l}\text { High rate of } \\
\text { adherence to } \\
\text { supplementation } \\
\text { (uptake of IFA or } \\
\text { MNP) observed in } \\
\text { both groups } \\
\text { (no statistical } \\
\text { difference) due to } \\
\text { home visits and } \\
\text { monitoring by } \\
\text { field workers }\end{array}$ \\
\hline $\begin{array}{l}\text { Rah, et al. } \\
\text { (2011) [19] }\end{array}$ & $\begin{array}{l}\text { Cross- } \\
\text { sectional } \\
\text { assessment } \\
\text { in } \\
\text { intervention } \\
\text { and control } \\
\text { areas }\end{array}$ & $\begin{array}{l}\text { I: } 358 \\
\text { C: } 361\end{array}$ & Lactating mothers & Bangladesh & $\begin{array}{l}\text { I: Micronutrient } \\
\text { powder + IFA } \\
\text { tablets + fortified } \\
\text { food ration + } \\
\text { social marketing } \\
\text { campaign + } \\
\text { education sessions } \\
\text { C: IFA tablets + } \\
\text { fortified } \\
\text { food ration }\end{array}$ & $\begin{array}{l}\text { No statistical difference in mean } \\
\text { weight, height, BMI, hemoglobin } \\
\text { levels, and anemia prevalence } \\
\text { between intervention or control } \\
\text { group, except proportion of thinness } \\
\text { and decreased anemia in women } \\
\text { consuming at least } 75 \% \text { sachets, } \\
\text { compared to those consuming }<75 \% \\
\text { sachets within the } \\
\text { intervention group. }\end{array}$ & $\begin{array}{l}\text { MNP distributors, } \\
\text { health and } \\
\text { nutrition staff of } \\
\text { local NGOs with } \\
\text { doctors, midwives, } \\
\text { nutritionists, } \\
\text { and volunteers }\end{array}$ & $\begin{array}{l}\text { Increased uptake } \\
\text { of MNP was } \\
\text { reported } \\
\text { (compliance) }\end{array}$ \\
\hline
\end{tabular}


Table 1. Cont

\begin{tabular}{|c|c|c|c|c|c|c|c|c|}
\hline Authors & $\begin{array}{l}\text { Study } \\
\text { Design }\end{array}$ & $\begin{array}{l}\text { Sample } \\
\text { Size }\end{array}$ & Study Population & $\begin{array}{l}\text { Location of the } \\
\text { Study }\end{array}$ & $\begin{array}{l}\text { Intervention/ } \\
\text { Comparison }\end{array}$ & Study Outcomes & $\begin{array}{c}\text { Delivery } \\
\text { Mechanism }\end{array}$ & $\begin{array}{c}\text { Change in } \\
\text { Coverage/Uptake }\end{array}$ \\
\hline $\begin{array}{l}\text { Ramakrishnan, } \\
\text { et al. (2012) } \\
{[20]}\end{array}$ & $\begin{array}{l}\text { Cross- } \\
\text { sectional } \\
\text { qualitative } \\
\text { research }\end{array}$ & $\begin{array}{l}\text { KII: } 31 \\
\text { FGD: } 35 \\
\text { IDI: } 15\end{array}$ & $\begin{array}{c}\text { Program officials, health } \\
\text { workers } \\
\text { (ASHA/ANM/AWW/ } \\
\text { Doctors) at the central } \\
\text { and peripheral level, } \\
\text { community leaders, and } \\
\text { volunteers. } \\
\text { Women } 18-45 \text { years. } \\
\text { with children } \leq 3 \text { years. }\end{array}$ & $\begin{array}{c}\text { Tamil Nadu and } \\
\text { Uttar Pradesh, } \\
\text { India }\end{array}$ & - & $\begin{array}{l}\text { Only } 27.6 \% \text { consumed } \geq 100 \text { IFA } \\
\text { tablets during their most recent } \\
\text { pregnancy, } 4 \% \text { took deworming } \\
\text { medicine during pregnancy, } 20.5 \% \\
\text { received supplementary food, and } \\
10.9 \% \text { received nutrition and health } \\
\text { education. } \\
\text { Barriers were lack of supply of } \\
\text { supplements, quality of food, } \\
\text { misconceptions concerning intake } \\
\text { of certain food products during } \\
\text { pregnancy, and in case of } \\
\text { take-home ration, sharing of food } \\
\text { with the household. }\end{array}$ & $\begin{array}{c}\text { Frontline workers } \\
\text { (ASHA, AWW, } \\
\text { ANM) }\end{array}$ & $\begin{array}{c}\text { Effective } \\
\text { counselling by } \\
\text { health workers } \\
\text { and targeted } \\
\text { media campaigns } \\
\text { improve uptake } \\
\text { (compliance) }\end{array}$ \\
\hline $\begin{array}{c}\text { Noznesky, } \\
\text { et al. (2012) } \\
{[21]}\end{array}$ & $\begin{array}{l}\text { Qualitative } \\
\text { research } \\
\text { using KII }\end{array}$ & KII: 48 & $\begin{array}{l}\text { Policy makers, program } \\
\text { managers, service } \\
\text { providers }\end{array}$ & Bihar, India & & $\begin{array}{l}\text { Only } 4 \% \text { consumed deworming } \\
\text { pill during their most recent } \\
\text { pregnancy, and } 0.6 \% \text { utilized } \\
\text { Anganwadi services. Barriers were } \\
\text { resource shortages, poverty, lack of } \\
\text { awareness, discrimination based on } \\
\text { socioeconomic status, as well as } \\
\text { policy-related barriers, such as lack } \\
\text { of focus on maternal nutrition, } \\
\text { knowledge level of the program } \\
\text { implementers about maternal } \\
\text { undernutrition, and a faulty } \\
\text { program management. }\end{array}$ & $\begin{array}{c}\text { Frontline workers } \\
\text { (ASHA, AWW, } \\
\text { ANM) }\end{array}$ & $\begin{array}{c}\text { Improved } \\
\text { essential inputs, } \\
\text { management } \\
\text { systems, and } \\
\text { reduced gender or } \\
\text { caste } \\
\text { discrimination }\end{array}$ \\
\hline
\end{tabular}


Table 1. Cont.

\begin{tabular}{|c|c|c|c|c|c|c|c|c|}
\hline Authors & $\begin{array}{l}\text { Study } \\
\text { Design }\end{array}$ & $\begin{array}{l}\text { Sample } \\
\text { Size }\end{array}$ & Study Population & $\begin{array}{l}\text { Location of the } \\
\text { Study }\end{array}$ & $\begin{array}{l}\text { Intervention/ } \\
\text { Comparison }\end{array}$ & Study Outcomes & $\begin{array}{c}\text { Delivery } \\
\text { Mechanism }\end{array}$ & $\begin{array}{c}\text { Change in } \\
\text { Coverage/Uptake }\end{array}$ \\
\hline $\begin{array}{l}\text { Nisar, et al. } \\
\text { (2014) [22] }\end{array}$ & $\begin{array}{l}\text { Secondary } \\
\text { data analysis: } \\
\text { NDHS } \\
(2006 / 2011) \\
\text { and PDHS } \\
(2006- \\
07 / 2012-13)\end{array}$ & $\begin{array}{l}\text { Pooled } \\
\text { NDHS: } \\
8196 \\
\text { mothers } \\
\text { Pooled } \\
\text { PDHS: } \\
\text { 13034 } \\
\text { mothers }\end{array}$ & Mothers & $\begin{array}{l}\text { Nepal and } \\
\text { Pakistan }\end{array}$ & $\begin{array}{c}\text { I: Any use of } \\
\text { IFA or }>90 \text { IFA } \\
\text { supplements } \\
\text { C: No use of } \\
\text { IFA }\end{array}$ & $\begin{array}{l}\text { AR of early neonatal deaths was } \\
\text { significantly reduced, by } 51 \% \text { in } \\
\text { Nepal and } 23 \% \text { in Pakistan, with } \\
\text { any use of IFA compared to none. } \\
\text { When IFA started at or before the } \\
\text { fifth month of pregnancy, the AR of } \\
\text { early neonatal mortality was } \\
\text { significantly reduced, by } 53 \% \text { in } \\
\text { Nepal and } 28 \% \text { in Pakistan, } \\
\text { compared to no IFA. When }>90 \text { IFA } \\
\text { supplements were used and started } \\
\text { at or before the fifth month, AR of } \\
\text { early neonatal deaths significantly } \\
\text { reduced, by } 57 \% \text { in Nepal and } 45 \% \\
\text { in Pakistan. }\end{array}$ & $\begin{array}{c}\text { Public-sector } \\
\text { facilities and CHW }\end{array}$ & $\begin{array}{l}\text { Training CHW, } \\
\text { making IFA } \\
\text { supplements } \\
\text { available for free, } \\
\text { and increasing } \\
\text { demands through } \\
\text { awareness- } \\
\text { promotion } \\
\text { programs }\end{array}$ \\
\hline $\begin{array}{l}\text { Vir, et al. } \\
\text { (2014) [23] }\end{array}$ & $\begin{array}{l}\text { Quasi experi- } \\
\text { mental } \\
\text { (mixed } \\
\text { methods } \\
\text { assessment) }\end{array}$ & $\begin{array}{l}\text { I: } 1825 \\
\text { C: } 1801\end{array}$ & $\begin{array}{c}\text { Mothers of children }<3 \\
\text { years }\end{array}$ & $\begin{array}{l}\text { Chhattisgarh, } \\
\text { India }\end{array}$ & $\begin{array}{l}\mathrm{I}^{*}: \mathrm{NSI}+ \\
\text { Mitanin } \\
\text { program } \\
\text { C: Only } \\
\text { Mitanin } \\
\text { program }\end{array}$ & $\begin{array}{l}\text { No significant difference in the } \\
\text { nutritional status of children } \\
\text { between two groups; more } \\
\text { households in the intervention } \\
\text { group than in the control group } \\
\text { had kitchen gardens } \\
(46.6 \% \text { vs. } 32.5 \%) .\end{array}$ & $\begin{array}{l}\text { Female CHWs } \\
\quad \text { (Mitanin) }\end{array}$ & $\begin{array}{l}\text { No significant } \\
\text { change in the } \\
\text { uptake of IFA } \\
\text { tablets but } \\
\text { significant } \\
\text { improvement in } \\
\text { coverage of three } \\
\text { ANC visits and } \\
\text { ANC within first } \\
\text { trimester }\end{array}$ \\
\hline $\begin{array}{c}\text { Gernand, } \\
\text { et al. (2015) } \\
\text { [24] }\end{array}$ & $\begin{array}{c}\text { Double- } \\
\text { blind, } \\
\text { cluster-RCT }\end{array}$ & $\begin{array}{l}\text { I: } 264 \\
\text { C: } 236\end{array}$ & Pregnant women & Bangladesh (rural) & $\begin{array}{l}\text { I: Multiple } \\
\text { micronutrient } \\
\text { powder } \\
\text { C: IFA }\end{array}$ & $\begin{array}{l}\text { No difference in maternal plasma } \\
\text { levels of hPL o PGH or cord plasma } \\
\text { levels of insulin, IGF-1, or IGFBP-1 } \\
\text { between two groups; however, } \\
\text { higher cord insulin concentration } \\
\text { was in women who were short and } \\
\text { higher hPL was found in women } \\
\text { carrying female fetuses. }\end{array}$ & $\begin{array}{l}\text { Local field } \\
\text { workers visited } \\
\text { weekly }\end{array}$ & $\begin{array}{c}\text { High compliance } \\
\text { with both MNP } \\
\text { and IFA noted in } \\
\text { the study }\end{array}$ \\
\hline
\end{tabular}


Table 1. Cont

\begin{tabular}{|c|c|c|c|c|c|c|c|c|}
\hline Authors & $\begin{array}{l}\text { Study } \\
\text { Design }\end{array}$ & $\begin{array}{l}\text { Sample } \\
\text { Size }\end{array}$ & Study Population & $\begin{array}{l}\text { Location of the } \\
\text { Study }\end{array}$ & $\begin{array}{l}\text { Intervention/ } \\
\text { Comparison }\end{array}$ & Study Outcomes & $\begin{array}{c}\text { Delivery } \\
\text { Mechanism }\end{array}$ & $\begin{array}{c}\text { Change in } \\
\text { Coverage/Uptake }\end{array}$ \\
\hline $\begin{array}{l}\text { Memon } \beta \text {, } \\
\text { et al. }(2015) \\
{[25]}\end{array}$ & $\begin{array}{l}\text { Exploratory } \\
\text { quasi- } \\
\text { experimental } \\
\text { design }\end{array}$ & $\begin{array}{l}\text { I: Pre } \\
(n=322) \\
\text { and Post } \\
(n=316) \\
\text { C: Pre }(n= \\
\text { 386) and } \\
\text { Post } \\
(n=361)\end{array}$ & Pregnant women & Northern Pakistan & $\begin{array}{c}\text { I: Community } \\
\text { mobilization + } \\
\text { education on } \\
\text { MNHC } \\
\text { through CHC } \\
\text { and group } \\
\text { sessions + } \\
\text { Routine health } \\
\text { services } \\
\text { C: Routine } \\
\text { health services }\end{array}$ & $\begin{array}{l}\text { Improvement in ANC, TT } \\
\text { vaccination, institutional delivery } \\
\text { rate, cord application, delayed } \\
\text { bathing, colostrum feeding, early } \\
\text { initiation of breastfeeding }(<1 \mathrm{~h} \text { of } \\
\text { birth) }(p<0.001) \text {, and reduction in } \\
\text { perinatal and neonatal mortality } \\
\text { rates }(p<0.05) \text { in the } \\
\text { intervention group. }\end{array}$ & $\begin{array}{l}\text { Female health } \\
\text { workers and CHW } \\
\text { conducted } \\
\text { monthly } \\
\text { household visits, } \\
\text { one-to-one } \\
\text { sessions, and } \\
\text { video sessions }\end{array}$ & $\begin{array}{c}\text { Increased uptake } \\
\text { of ANC visits and } \\
\text { care }\end{array}$ \\
\hline $\begin{array}{c}\text { Sablok, et al. } \\
\text { (2015) [26] }\end{array}$ & $\mathrm{RCT}$ & $\begin{array}{l}\text { I: } 120 \\
\text { C: } 60\end{array}$ & Pregnant women & Delhi, India & $\begin{array}{l}\text { I: Vit D Supple- } \\
\text { mentation } \\
\text { C: No supple- } \\
\text { mentation of } \\
\quad \text { Vit D }\end{array}$ & $\begin{array}{l}\text { Intervention group had lower } \\
\text { incidence of preterm labour } \\
(p=0.02) \text {; higher number of } \\
\text { newborns to mothers in the control } \\
\text { group had lower Vit D levels } \\
(<25 \mathrm{nmol} / \mathrm{L})(p<0.001) \text { and lower } \\
\text { mean birth weight; and higher } \\
\text { proportion of SGA in control group } \\
\qquad(p=0.04) .\end{array}$ & $\begin{array}{l}\text { Unclear } \\
\text { (Department of } \\
\text { Obstetrics and } \\
\text { Gynecology, } \\
\text { Tertiary hospital, } \\
\text { Delhi) }\end{array}$ & Not reported \\
\hline $\begin{array}{l}\text { Kosec, et al. } \\
\text { (2015) [27] }\end{array}$ & $\begin{array}{c}\text { Secondary } \\
\text { data analysis } \\
\text { of DLHS and } \\
\text { facility } \\
\text { workers } \\
\text { Surveys 2012 }\end{array}$ & $\begin{array}{l}6002 \text { house- } \\
\text { holds in } \\
400 \\
\text { villages }\end{array}$ & $\begin{array}{l}\text { Household and } \\
\text { frontline workers } \\
(\mathrm{ASHA}, \mathrm{AWW})\end{array}$ & Bihar, India & - & $\begin{array}{l}\text { Monetary incentives for AWW are } \\
\text { strong predictor of receipt of } \\
\text { immunization services ( } 0-2 \text { years }) \\
\text { and households receiving general } \\
\text { nutrition information. }\end{array}$ & $\begin{array}{c}\text { ASHA and AWW } \\
\text { delivering routine } \\
\text { services }\end{array}$ & $\begin{array}{l}\text { Incentivizing } \\
\text { frontline workers } \\
\text { and improving } \\
\text { performance } \\
\text { increase service } \\
\text { uptake by } \\
\text { households }\end{array}$ \\
\hline $\begin{array}{l}\text { Jolly, et al. } \\
\text { (2016) [28] }\end{array}$ & $\begin{array}{c}\text { Cross- } \\
\text { sectional } \\
\text { comparative } \\
\text { study }\end{array}$ & $\begin{array}{l}\text { I: } 607 \\
\text { C: } 599\end{array}$ & Married women & $\begin{array}{l}\text { Bangladesh } \\
\text { (Urban slums) }\end{array}$ & $\begin{array}{l}\text { I: MANOSHI II } \\
\text { program } \\
\text { C: Without } \\
\text { MANOSHI } \\
\text { services }\end{array}$ & $\begin{array}{l}\text { Increased odds of improved } \\
\text { maternal-health service indicators } \\
\text { (4 or more ANC visits, receipt of } \\
\text { IFA and TT injection, PNC within } \\
48 \text { h of birth, and institutional } \\
\text { delivery rate) in the intervention } \\
\text { group }(p<0.05) .\end{array}$ & $\begin{array}{c}\text { Female CHW } \\
\text { doing household } \\
\text { visits }\end{array}$ & $\begin{array}{l}\text { Uptake of four or } \\
\text { more ANC } \\
\text { checkups, } \\
\text { institutional } \\
\text { delivery, skilled } \\
\text { assisted delivery, } \\
\text { and PNC } \\
\text { increased in the } \\
\text { intervention group }\end{array}$ \\
\hline
\end{tabular}


Table 1. Cont

\begin{tabular}{|c|c|c|c|c|c|c|c|c|}
\hline Authors & $\begin{array}{l}\text { Study } \\
\text { Design }\end{array}$ & $\begin{array}{l}\text { Sample } \\
\text { Size }\end{array}$ & Study Population & $\begin{array}{l}\text { Location of the } \\
\text { Study }\end{array}$ & $\begin{array}{l}\text { Intervention/ } \\
\text { Comparison }\end{array}$ & Study Outcomes & $\begin{array}{c}\text { Delivery } \\
\text { Mechanism }\end{array}$ & $\begin{array}{c}\text { Change in } \\
\text { Coverage/Uptake }\end{array}$ \\
\hline $\begin{array}{c}\text { Kadiyala, } \\
\text { et al. (2016) } \\
\text { [29] }\end{array}$ & $\begin{array}{l}\text { A case study } \\
\text { of digital } \\
\text { green } \\
\text { approach } \\
\text { (qualitative } \\
\text { assessment) }\end{array}$ & $\begin{array}{l}* * \text { I: IDI: } 72 \\
\text { SSI: } 73 \\
\text { KII: } 6\end{array}$ & $\begin{array}{l}\text { SHG members (PLW, } \\
\text { CFM, mothers of } \\
\text { adolescent girls, and } \\
\text { other women); MIL, } \\
\text { husbands, FLW, key } \\
\text { stakeholders, } \\
\text { protagonists, FLPP * }\end{array}$ & Odisha, India & $\begin{array}{l}\text { I: MIYCN BCC + } \\
\text { Digital green } \\
\text { approach to } \\
\text { agriculture } \\
\text { extension }\end{array}$ & $\begin{array}{l}\text { Intervention well received by } \\
\text { rural communities and viewed } \\
\text { as complementary to routine } \\
\text { services; intervention was } \\
\text { perceived as a credible source } \\
\text { of information related to health } \\
\text { and nutrition. }\end{array}$ & CHWs & $\begin{array}{c}\text { Participatory, } \\
\text { dialogue-based } \\
\text { interventions with } \\
\text { women's groups, } \\
\text { as well as video } \\
\text { education, } \\
\text { improved } \\
\text { maternal and } \\
\text { childcare services }\end{array}$ \\
\hline $\begin{array}{l}\text { Mridha, et al. } \\
\text { (2016) [30] }\end{array}$ & $\begin{array}{l}\text { Researcher- } \\
\quad \text { blind, } \\
\text { longitudinal, } \\
\quad \text { cluster- } \\
\text { randomized } \\
\text { effectiveness } \\
\quad \text { trial } \\
\end{array}$ & $\begin{array}{l}\text { I: } 1047 \\
\text { C: } 2964\end{array}$ & $\begin{array}{l}\text { Pregnant women } \leq 20 \\
\text { gestational weeks }\end{array}$ & Bangladesh (rural) & $\begin{array}{l}\text { I: -Mothers given } \\
\text { LNS-PLs } \\
\text { C: Mother given } \\
\text { IFA }\end{array}$ & $\begin{array}{l}\text { Increased mean birth weight, } \\
\text { WAZ, birth length, LAZ, head } \\
\text { circumference, HCZ, BMIZ in } \\
\text { the children born to mothers in } \\
\text { the intervention group }\end{array}$ & NGO staff & $\begin{array}{l}\text { Adherence to IFA } \\
\text { was more than the } \\
\text { intervention }\end{array}$ \\
\hline
\end{tabular}


Table 1. Cont.

\begin{tabular}{|c|c|c|c|c|c|c|c|c|}
\hline Authors & $\begin{array}{l}\text { Study } \\
\text { Design }\end{array}$ & $\begin{array}{l}\text { Sample } \\
\text { Size }\end{array}$ & Study Population & $\begin{array}{l}\text { Location of the } \\
\text { Study }\end{array}$ & $\begin{array}{l}\text { Intervention/ } \\
\text { Comparison }\end{array}$ & Study Outcomes & $\begin{array}{c}\text { Delivery } \\
\text { Mechanism }\end{array}$ & $\begin{array}{c}\text { Change in } \\
\text { Coverage/Uptake }\end{array}$ \\
\hline $\begin{array}{c}\text { Nguyen, } \\
\text { et al. (2017) } \\
\text { [32] }\end{array}$ & $\begin{array}{l}\text { Cross- } \\
\text { sectional } \\
\text { study }\end{array}$ & $\begin{array}{l}\text { Pregnant } \\
\text { women } \\
(n=600) ; \\
\text { Recently } \\
\text { delivered } \\
(n=2000)\end{array}$ & $\begin{array}{l}\text { Pregnant women and } \\
\text { recently delivered }\end{array}$ & Bangladesh & $\begin{array}{c}\text { I: Standard } \\
\text { nutrition } \\
\text { intervention of } \\
\text { nutrition } \\
\text { education + IFA + } \\
\text { and calcium } \\
\text { supplementation + } \\
\text { deworming + } \\
\text { IYCF counseling }\end{array}$ & $\begin{array}{l}\text { Good nutrition knowledge, } \\
\text { women's self-efficacy, } \\
\text { perception of enabling social } \\
\text { norms, high husband's support, } \\
\text { early and more prenatal visits, } \\
\text { provision of free supplements } \\
\text { improve maternal nutrition } \\
\text { practices (IFA and calcium } \\
\text { intake and diverse diets). }\end{array}$ & $\begin{array}{l}\text { Frontline health } \\
\text { workers } \\
\text { conducted } \\
\text { monthly home } \\
\text { visits }\end{array}$ & $\begin{array}{l}\text { Observed increase } \\
\text { in the uptake of } \\
\text { IFA/calcium } \\
\text { tablets and four or } \\
\text { more prenatal } \\
\text { visits }\end{array}$ \\
\hline
\end{tabular}

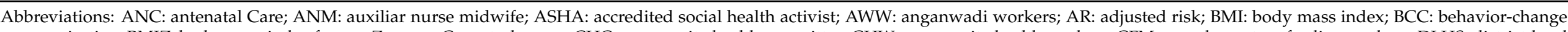

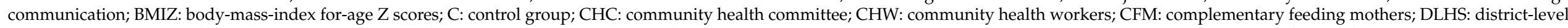

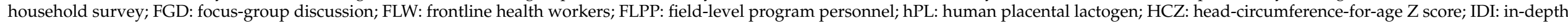

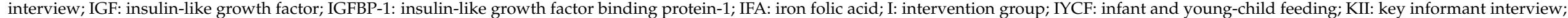

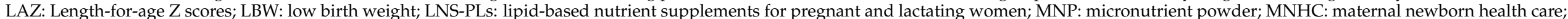
Pre

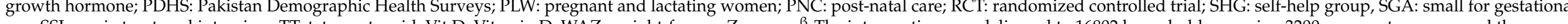

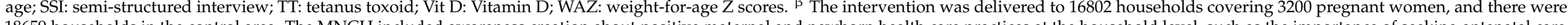

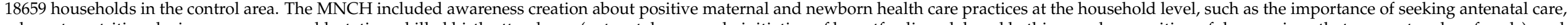

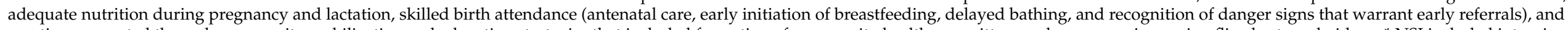

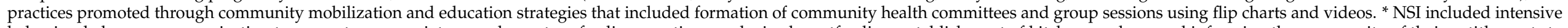

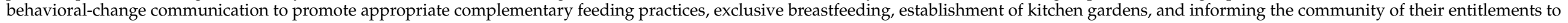

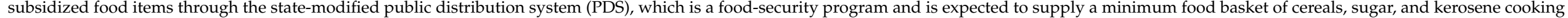

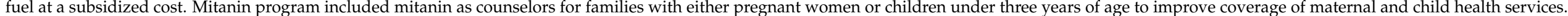

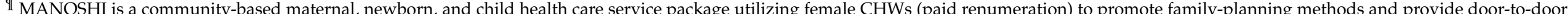

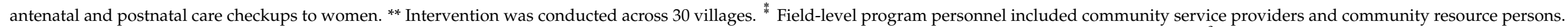

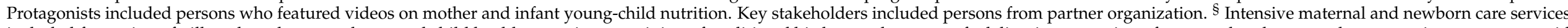

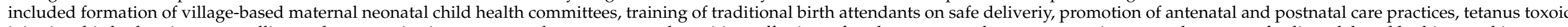

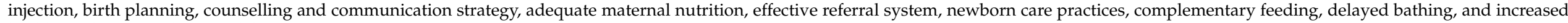
health-workers attendance at delivery. 
Table 2. Characteristics of the included studies $(n=15)$.

\begin{tabular}{|c|c|c|c|c|c|c|c|c|}
\hline Authors & Study Design & Sample Size & Study Population & $\begin{array}{l}\text { Location of } \\
\text { the Study }\end{array}$ & $\begin{array}{l}\text { Intervention/ } \\
\text { Comparison }\end{array}$ & Study Outcomes & $\begin{array}{c}\text { Delivery } \\
\text { Mechanism }\end{array}$ & $\begin{array}{c}\text { Change in } \\
\text { Coverage/Uptake }\end{array}$ \\
\hline $\begin{array}{l}\text { Nguyen, et al. } \\
\text { (2017) [33] }\end{array}$ & $\begin{array}{l}\text { Cluster RCT with } \\
\text { cross-sectional } \\
\text { baseline (2015) } \\
\text { and endline (2016) } \\
\text { survey }\end{array}$ & $\begin{array}{l}\text { I: PW: } 300 \\
\text { RDW: } 1000 \\
\text { C: PW: } 300 \\
\text { RDW: } 1000\end{array}$ & $\begin{array}{l}\text { Pregnant women } \\
\text { and } \\
\text { recently delivered } \\
\text { women }\end{array}$ & Bangladesh & $\begin{array}{c}\text { I: } \\
\text { nutrition-focused } \\
\mathbb{I I}_{\mathrm{MNCH}} \\
\text { intervention } \\
\mathrm{C} \text { : standard } \\
\text { MNCH } \\
\text { intervention }\end{array}$ & $\begin{array}{l}\text { Increase in consumption } \\
\text { of IFA and calcium } \\
\text { supplements, } \geq 5 \text { food } \\
\text { groups, and most macro } \\
\text { and micronutrients; } \\
\text { increase in individual } \\
\text { food groups consumed } \\
\text { among women; increase } \\
\text { in EBF by women }\end{array}$ & $\begin{array}{l}\text { Salaried health } \\
\text { workers and } \\
\text { community health } \\
\text { volunteers } \\
\text { conducted } \\
\text { monthly home } \\
\text { visits }\end{array}$ & $\begin{array}{c}\text { Increased } \\
\text { probability of } \\
\text { early ANC visits } \\
\text { and receipt of free } \\
\text { iron and calcium } \\
\text { tablets in the } \\
\text { intervention group }\end{array}$ \\
\hline $\begin{array}{c}\text { Raghunathan, } \\
\text { et al. (2017) } \\
\text { [34] }\end{array}$ & $\begin{array}{l}\text { Cross-sectional } \\
\text { study }\end{array}$ & $\begin{array}{l}\text { I: } 534 \\
\text { C: } 627\end{array}$ & $\begin{array}{l}\text { Pregnant and } \\
\text { lactating women }\end{array}$ & Odisha, India & $\begin{array}{l}\text { I: women who } \\
\text { received money } \\
\text { under CCT } \\
\text { scheme } \\
\text { C: women who } \\
\text { did not receive } \\
\text { money under CCT } \\
\text { scheme }\end{array}$ & $\begin{array}{l}\text { Increase in likelihood of } \\
\text { pregnancy registration, } \\
\text { receiving ANC services } \\
(5 \mathrm{pp}) \text {, and IFA tablets } \\
(10 \mathrm{pp}) \text { and a decline of } \\
0.84 \text { on the household } \\
\text { food insecurity } \\
\text { assessment scale }\end{array}$ & $\begin{array}{l}\text { DBT by the } \\
\text { government and } \\
\text { essential nutrition } \\
\text { intervention by } \\
\text { AWW and ASHA }\end{array}$ & $\begin{array}{l}\text { CCT scheme } \\
\text { increased the } \\
\text { coverage of ANC } \\
\text { services, IFA } \\
\text { consumption, and } \\
\text { pregnancy } \\
\text { registration }\end{array}$ \\
\hline $\begin{array}{l}\text { Dewey, et al. } \\
\text { (2017) [35] }\end{array}$ & $\begin{array}{l}\text { Researcher-blind, } \\
\text { longitudinal, } \\
\text { cluster- } \\
\text { randomized } \\
\text { effectiveness trial }\end{array}$ & $\begin{array}{l}\alpha \text { IFA-MNP = 1052; } \\
\text { IFA-LNS = 930; } \\
\text { LNS-LNS = 1047; } \\
\text { Control (IFA) = } 982\end{array}$ & $\begin{array}{l}\text { Pregnant women } \\
\text { at } \leq 20 \text { gestational } \\
\text { age and children }\end{array}$ & Bangladesh & $\begin{array}{l}\alpha \text { I: IFA-MNP: } \\
\text { mother given IFA } \\
\text { and child given } \\
\text { MNP; IFA-LNS: } \\
\text { mother given IFA } \\
\text { and child LNS; } \\
\text { LNS-LNS: mother } \\
\text { and child both } \\
\text { given LNS; } \\
\text { C: IFA-control: } \\
\text { mother given IFA } \\
\text { and child given } \\
\text { nothing }\end{array}$ & $\begin{array}{l}\text { LNS-LNS group had } \\
\text { significantly higher } \\
\text { LAZ (+0.13 compared } \\
\text { with the IFA-MNP group) } \\
\text { and head circumference } \\
\text { (+0.15 z score compared } \\
\text { with the IFA-Control } \\
\text { group); stunting } \\
\text { prevalence (LAZ }<-2) \\
\text { was lower in the } \\
\text { LNS-LNS group at } \\
18 \text { months than in the } \\
\text { IFA-MNP group (OR: } 0.70 ; \\
\text { 95\% CI: } 0.53,0.92), \text { but the } \\
\text { difference diminished by } \\
24 \text { months (OR: } 0.81 ; 95 \% \\
\text { CI: } 0.63,1.04)\end{array}$ & NGO staff & $\begin{array}{l}\text { Adherence to the } \\
\text { interventions was } \\
\text { reported but not to } \\
\text { other services, } \\
\text { which was higher } \\
\text { in LNS-LNS and } \\
\text { IFA-LNS than } \\
\text { LNS-MNP }\end{array}$ \\
\hline
\end{tabular}


Table 2. Cont.

\begin{tabular}{|c|c|c|c|c|c|c|c|c|}
\hline Authors & Study Design & Sample Size & Study Population & $\begin{array}{l}\text { Location of } \\
\text { the Study }\end{array}$ & $\begin{array}{l}\text { Intervention/ } \\
\text { Comparison }\end{array}$ & Study Outcomes & $\begin{array}{c}\text { Delivery } \\
\text { Mechanism }\end{array}$ & $\begin{array}{c}\text { Change in } \\
\text { Coverage/Uptake }\end{array}$ \\
\hline $\begin{array}{l}\text { Nair, et al. } \\
\text { (2017) [36] }\end{array}$ & Cluster RCT & $\begin{array}{l}\text { I: PW: } 2805 ; \\
\text { Infants: } 1460 \\
\text { C: PW: } 2952 ; \\
\text { Infants: } 1541\end{array}$ & $\begin{array}{l}\text { Pregnant women } \\
\text { and infants }\end{array}$ & $\begin{array}{l}\text { Jharkhand and } \\
\text { Odisha, India }\end{array}$ & $\begin{array}{l}\text { I: Single home } \\
\text { visit during 3rd } \\
\text { trimester, monthly } \\
\text { home visit to } \\
\text { children }<2 \text { years } \\
\text { for counseling and } \\
\text { growth promotion, } \\
\text { 2-3 participatory } \\
\text { meetings with } \\
\text { local women's } \\
\text { groups }\end{array}$ & $\begin{array}{l}\text { No significant effect on } \\
\text { EBF, timely initiation of } \\
\text { complementary feeding, } \\
\text { morbidity, appropriate } \\
\text { home care, or } \\
\text { care-seeking during } \\
\text { childhood illnesses of the } \\
\text { intervention; more } \\
\text { pregnant women and } \\
\text { children attained MDD, } \\
\text { more mothers washed } \\
\text { their hands before feeding } \\
\text { children, fewer children } \\
\text { were underweight at } 18 \\
\text { months, and fewer } \\
\text { infants died }\end{array}$ & $\begin{array}{l}\text { Community- } \\
\text { based incentivized } \\
\text { volunteers }\end{array}$ & $\begin{array}{c}\text { No significant } \\
\text { change in the } \\
\text { uptake of } \\
\text { maternal or } \\
\text { childcare services }\end{array}$ \\
\hline
\end{tabular}


Table 2. Cont.

\begin{tabular}{|c|c|c|c|c|c|c|c|c|}
\hline Authors & Study Design & Sample Size & Study Population & $\begin{array}{l}\text { Location of } \\
\text { the Study }\end{array}$ & $\begin{array}{l}\text { Intervention/ } \\
\text { Comparison }\end{array}$ & Study Outcomes & $\begin{array}{c}\text { Delivery } \\
\text { Mechanism }\end{array}$ & $\begin{array}{c}\text { Change in } \\
\text { Coverage/Uptake }\end{array}$ \\
\hline $\begin{array}{l}\text { Hashmi, et al. } \\
\text { (2018) [38] }\end{array}$ & $\begin{array}{l}\text { Convergent } \\
\text { parallel } \\
\text { mixed-method } \\
\text { design }\end{array}$ & $\begin{array}{l}\text { Cross sectional } \\
\text { survey = } 388 \text { PW } \\
\text { FGD = } 11 \text { women } \\
\text { IDI = } 4 \text { midwives }\end{array}$ & Pregnant women & Thailand & - & $\begin{array}{l}\text { A high proportion of } \\
\text { women had limited } \\
\text { knowledge of and } \\
\text { poor dietary practices. } \\
\text { Sweetened-drink } \\
\text { consumption in the last } \\
24 \text { h, as well as being } \\
\text { non-teenaged } \\
\text { multigravida woman, } \\
\text { significantly associated } \\
\text { with high BMI compared } \\
\text { to normal BMI }\end{array}$ & - & $\begin{array}{c}\text { Proportion of first } \\
\text { antenatal care visit } \\
\text { higher } \\
\text { for the first } \\
\text { trimester than in } \\
\text { the second or third } \\
\text { trimesters }\end{array}$ \\
\hline $\begin{array}{l}\text { Khanam, et al. } \\
\text { (2018) [39] }\end{array}$ & $\begin{array}{l}\text { Retrospective } \\
\text { cohort design }\end{array}$ & $\begin{array}{c}\text { Case: Women who } \\
\text { had PIH } \\
\text { Control: Women } \\
\text { did not develop } \\
\text { PIH }\end{array}$ & Pregnant women & Bangladesh & I: MNI program & $\begin{array}{l}\text { Women who consumed } \\
500 \mathrm{mg} / \mathrm{d} \text { calcium tablets } \\
\text { for more than } 6 \text { months } \\
\text { during pregnancy had a } \\
45 \% \text { lower risk of } \\
\text { developing hypertension } \\
\text { compared to those who } \\
\text { consumed less calcium } \\
\text { (RR }=0.55,95 \% \mathrm{CI}= \\
0.33-0.93\end{array}$ & $\mathrm{CHW}$ & $\begin{array}{c}\text { No significant } \\
\text { difference in the } \\
\text { covergae of four or } \\
\text { more ANC visits } \\
\text { in PIH or non-PIH } \\
\text { women }\end{array}$ \\
\hline $\begin{array}{l}\text { Saville, et al. } \\
\text { (2018) [40] }\end{array}$ & $\begin{array}{l}\text { Four-arm cluster } \\
\text { RCT }\end{array}$ & $\begin{array}{c}\text { I: PLA + food: } \\
\text { 2997; } \\
\text { PLA +cash: 3065; } \\
\text { PLA only: } 2448 ; \\
\text { C: } 2426\end{array}$ & Pregnant women & Nepal & $\begin{array}{c}\text { I: Arm 1: PLA only } \\
\text { Arm 2: PLA + } \\
\text { food supplement } \\
\text { Arm 3: PLA + cash } \\
\text { transfer } \\
\text { C: current gov't } \\
\text { program }\end{array}$ & $\begin{array}{l}\text { Compared to the control } \\
\text { arm, mean BW } \\
\text { significantly higher in the } \\
\text { PLA + food arm, by } 78.0 \mathrm{~g} \\
(95 \% \text { CI } 13.9,142.0) \text { and } \\
\text { not in others; no } \\
\text { significant difference in } \\
\text { any other outcome (WAZ, } \\
\text { LAZ, WLZ, HC, maternal } \\
\text { BMI, MUAC, and IYCF) }\end{array}$ & $\begin{array}{c}\text { Female } \\
\text { community health } \\
\text { volunteers + } \\
\text { nutrition } \\
\text { mobilizers } \\
\text { (incentivized) }\end{array}$ & $\begin{array}{c}\text { Enhanced } \\
\text { participation in } \\
\text { women's groups } \\
\text { increased } \\
\text { institutional } \\
\text { delivery rate in the } \\
\text { intervention group }\end{array}$ \\
\hline
\end{tabular}


Table 2. Cont.

\begin{tabular}{|c|c|c|c|c|c|c|c|c|}
\hline Authors & Study Design & Sample Size & $\begin{array}{c}\text { Study } \\
\text { Population }\end{array}$ & $\begin{array}{l}\text { Location of } \\
\text { the Study }\end{array}$ & $\begin{array}{l}\text { Intervention/ } \\
\text { Comparison }\end{array}$ & Study Outcomes & $\begin{array}{c}\text { Delivery } \\
\text { Mechanism }\end{array}$ & $\begin{array}{c}\text { Change in } \\
\text { Coverage/Uptake }\end{array}$ \\
\hline $\begin{array}{l}\text { Stevens, } \\
\text { et al. (2018) } \\
\quad[42]\end{array}$ & $\begin{array}{l}\text { Mixed-method, } \\
\text { cross sectional } \\
\text { survey and } \\
\text { qualitative study } \\
\text { design }\end{array}$ & $\begin{array}{c}\text { Pregnant } \\
\text { Women: } \\
\text { baseline = 371, } \\
\text { end line }=307 \\
\text { Local Health } \\
\text { Workers: } \\
\text { baseline }=100, \\
\text { end line }=79\end{array}$ & $\begin{array}{l}\text { Pregnant } \\
\text { women and } \\
\text { local health } \\
\text { workers }\end{array}$ & $\begin{array}{l}\text { Thailand- } \\
\text { Myanmar }\end{array}$ & $\begin{array}{c}\text { I: } \\
\text { community-based } \\
\text { participatory } \\
\text { action plan } \\
\text { (workshops for } \\
\text { health workers + } \\
\text { posters in centers + } \\
\text { pamphlets } \\
\text { distribution + } \\
\text { presentations + } \\
\text { small group } \\
\text { discussions) }\end{array}$ & $\begin{array}{l}\text { No significant improvement in } \\
\text { preconception folic acid uptake; } \\
\text { however, substantial increase in local } \\
\text { healthcare workers' knowledge }\end{array}$ & $\begin{array}{l}\text { Local health } \\
\text { workers (medics, } \\
\text { midwives, nurses } \\
\text { + ultrasound } \\
\text { workers + basic } \\
\text { healthcare } \\
\text { workers) }\end{array}$ & $\begin{array}{l}\text { No significant } \\
\text { uptake of } \\
\text { preconception } \\
\text { folic acid }\end{array}$ \\
\hline $\begin{array}{l}\text { Stevens, } \\
\text { et al. (2018) } \\
{[43]}\end{array}$ & $\begin{array}{l}\text { Village-matched } \\
\text { cluster RCT (3rd } \\
\text { phase of a } \\
\text { multiphase RCT) }\end{array}$ & $\begin{array}{l}\text { I: } 58 \\
\text { C: } 29\end{array}$ & $\begin{array}{l}\text { Undernourished } \\
\text { pregnant } \\
\text { women with } \\
\text { MUAC of } \\
\leq 22.1 \mathrm{~cm}\end{array}$ & $\begin{array}{l}\text { Northern } \\
\text { Bangladesh } \\
\quad \text { (rural) }\end{array}$ & $\begin{array}{c}\text { I: nutrition } \\
\text { screening + } \\
\text { nutrition } \\
\text { education + } \\
\text { ANC/PNC } \\
\text { services + } \\
\text { supplements } \\
\text { C: nutrition } \\
\text { screening + } \\
\text { nutrition } \\
\text { education + } \\
\text { ANC/PNC } \\
\text { services }\end{array}$ & $\begin{array}{c}\text { MUAC significantly larger in infants } \\
\text { of mothers in the intervention group } \\
\text { compared to control group at } \\
6 \text { months } \\
(p<0.05) \text {. Mean BW in babies of } \\
\text { supplemented mothers (mean: } \\
2.91 \mathrm{~kg} \text {; SD: } 0.19 \text { ) higher than in } \\
\text { babies of mothers in control group } \\
\text { (mean: } 2.72 \mathrm{~kg} \text {; SD: } 0.13 \text { ); proportion } \\
\text { of LBW babies in the } \\
\text { intervention group } \\
\text { was much lower (event rate }=0.04 \text { ) } \\
\text { than in the control group } \\
\text { (event rate }=0.16 \text { ). However, none of } \\
\text { these differences are } \\
\text { statistically significant } \\
\text { ( } p>0.05 \text { ), most likely due to small } \\
\text { sample size. The intervention } \\
\text { reduced the risk of wasting } \\
\text { at } 6 \text { months by } 63.38 \% \text { (RRR = } 0.6338 \text { ) } \\
\text { and of low birth weight by } 88.58 \% \\
\text { (RRR = 0.8858), with NNT of } 2.22 \\
\text { and } 6.32, \text { respectively. }\end{array}$ & $\begin{array}{c}\text { Female } \\
\text { community } \\
\text { nutrition } \\
\text { volunteers and } \\
\text { one male and one } \\
\text { female supervisor }\end{array}$ & $\begin{array}{l}\text { Higher } \\
\text { registration of } \\
\text { women within the } \\
\text { first trimester }\end{array}$ \\
\hline
\end{tabular}


Table 2. Cont.

\begin{tabular}{|c|c|c|c|c|c|c|c|c|}
\hline Authors & Study Design & Sample Size & Study Population & $\begin{array}{l}\text { Location of } \\
\text { the Study }\end{array}$ & $\begin{array}{l}\text { Intervention/ } \\
\text { Comparison }\end{array}$ & Study Outcomes & $\begin{array}{c}\text { Delivery } \\
\text { Mechanism }\end{array}$ & $\begin{array}{l}\text { Change in } \\
\text { Cover- } \\
\text { age/Uptake }\end{array}$ \\
\hline $\begin{array}{l}\text { Svefors, et al. } \\
\text { (2018) [44] }\end{array}$ & $\begin{array}{l}\text { Factorial } \\
\text { randomized } \\
\text { trial (Nov 2001 } \\
\text { to Feb 2009) }\end{array}$ & $\begin{array}{c}\text { E60Fe: 738, EMMS: } \\
\text { 740, E30Fe: 739, } \\
\text { U60Fe: 741, } \\
\text { UMMS: 741, } \\
\text { U30Fe: } 741\end{array}$ & Pregnant women & $\begin{array}{l}\text { Bangladesh } \\
\text { (rural) }\end{array}$ & $\begin{array}{l}\text { I: MINIMat trial of } \\
\text { food } \\
\text { supplementation }{ }^{*} \\
\text { (E30Fe, E60Fe, } \\
\text { EMMS, U30Fe, } \\
\text { U60Fe, UMMS) }\end{array}$ & $\begin{array}{l}\text { By incremental U60Fe to EMMS, one } \\
\text { disability adjusted life years, averted at a } \\
\text { cost of USD } 24\end{array}$ & $\begin{array}{l}\text { Community } \\
\text { volunteers }\end{array}$ & Not reported \\
\hline $\begin{array}{l}\text { Wendt, et al. } \\
\text { (2018) [45] }\end{array}$ & $\begin{array}{l}\text { Cross- } \\
\text { sectional, } \\
\text { observational, } \\
\text { mixed-method } \\
\text { (Nov 2011 to } \\
\text { July 2012) }\end{array}$ & $\begin{array}{c}\text { IDI: } 59 \text { (health } \\
\text { workers at state, } \\
\text { district, block, } \\
\text { health sub-centre, } \\
\text { and village levels) } \\
\text { ANM survey: } 340\end{array}$ & $\begin{array}{l}\text { Health workers at } \\
\text { state, district, } \\
\text { block, health } \\
\text { sub-centre, } \\
\text { and village levels; } \\
\text { ANM }\end{array}$ & Bihar, India & - & $\begin{array}{l}\text { 44\% of ANM were out of IFA stock. Stock } \\
\text { levels and supply-chain practices varied } \\
\text { greatly across districts. Specific bottlenecks } \\
\text { impacting IFA were forecasting, } \\
\text { procurement, storage, disposal, lack of } \\
\text { personnel, and few training opportunities } \\
\text { for key players in the supply chain }\end{array}$ & $\begin{array}{c}\text { ASHA, } \\
\text { AWW, ANM }\end{array}$ & Not reported \\
\hline $\begin{array}{l}\text { Pavithra, } \\
\text { et al. (2019) } \\
{[46]}\end{array}$ & $\begin{array}{l}\text { Community- } \\
\text { based } \\
\text { intervention } \\
\text { study } \\
\text { (December } \\
2012 \text { to } \\
\text { October 2014) }\end{array}$ & $\begin{array}{l}\text { I: } 64 \text { children } \\
\text { C: } 64 \text { children }\end{array}$ & $\begin{array}{c}57 \text { mothers and } 60 \\
\text { mothers of } 64 \\
\text { moderate and } \\
\text { severely } \\
\text { malnourished } \\
\text { children aged } \\
13-60 \text { months in } \\
\text { the intervention } \\
\text { group and control } \\
\text { group, } \\
\text { respectively }\end{array}$ & $\begin{array}{l}\text { Puducherry, } \\
\text { India (rural) }\end{array}$ & $\begin{array}{l}\text { I: one-to-one } \\
\text { communication } \\
\text { with mothers } \\
\text { concerning their } \\
\text { child's nutritional } \\
\text { status and growth } \\
\text { monitoring; } \\
\text { education and } \\
\text { child feeding } \\
\text { practices; } \\
\text { reinforcement of } \\
\text { contents of health } \\
\text { education }\end{array}$ & $\begin{array}{l}\text { Awareness level in all domains increased } \\
\text { significantly in the intervention group; } \\
81 \% \text { (52) of malnourished children turned } \\
\text { out normal, whereas in the control group, } \\
64 \% \text { ( } 41 \text { ) } \\
\text { of became normal; statistically significant } \\
\text { difference between the mean changes in } \\
\text { protein intake among boys ( } 15.34 \mathrm{~g} \text { to } \\
19.91 \mathrm{~g} \text { in the intervention group against } \\
13.6 \mathrm{~g} \text { to } 16.24 \mathrm{~g} \text { in the control group) and } \\
\text { girls (15.09 g to } 19.57 \mathrm{~g} \text { in the intervention } \\
\text { group against } \\
13.36 \mathrm{~g} \text { to } 16.51 \mathrm{~g} \text { in the control group), as } \\
\text { well as calorie intake among girls } \\
\text { ( } 993.86 \text { kcal to } 1116.55 \mathrm{kcal} \text { in the } \\
\text { intervention group against } 992.65 \\
\text { kcal to } 1078.75 \mathrm{kcal} \text { in the control group) } \\
\text { between the two groups }\end{array}$ & Unclear & Not reported \\
\hline
\end{tabular}


Table 2. Cont

\begin{tabular}{|c|c|c|c|c|c|c|c|c|}
\hline Authors & Study Design & Sample Size & Study Population & $\begin{array}{l}\text { Location of } \\
\text { the Study }\end{array}$ & $\begin{array}{l}\text { Intervention/ } \\
\text { Comparison }\end{array}$ & Study Outcomes & $\begin{array}{c}\text { Delivery } \\
\text { Mechanism }\end{array}$ & $\begin{array}{l}\text { Change in } \\
\text { Cover- } \\
\text { age/Uptake }\end{array}$ \\
\hline $\begin{array}{c}\text { Dhaded, } \\
\text { et al. (2020) } \\
{[47]}\end{array}$ & $\begin{array}{l}\text { Secondary } \\
\text { analysis; the } \\
\text { parent study } \\
\text { was an } \\
\text { individually } \\
\text { randomized, } \\
\text { non-masked, } \\
\text { multi-site } \\
\text { randomized } \\
\text { controlled } \\
\text { efficacy trial }\end{array}$ & $\begin{array}{l}\text { I: LBM-PC: } 1281 ; \\
\text { LBM-FT: } 1277 \\
\text { C: } 1280\end{array}$ & $\begin{array}{c}\text { Mothers and their } \\
\text { children } \\
\text { (newborns) }\end{array}$ & $\begin{array}{l}\text { India and } \\
\text { Pakistan } \\
\text { (rural) }\end{array}$ & $\begin{array}{l}\text { I: Arm 1: received } \\
\text { LBM at least } \\
\text { 3-months prior to } \\
\text { conception; } \\
\text { Arm 2: received } \\
\text { LBM near the end } \\
\text { of the 1st trimester; } \\
\text { additional } \\
\text { protein-energy } \\
\text { supplement was } \\
\text { given to women } \\
\text { whose BMI was } \\
<20 \mathrm{~kg} / \mathrm{m}^{2} \text { for } \\
\text { both Arm } 1 \& 2 \text { till } \\
\text { delivery }\end{array}$ & $\begin{array}{l}\text { LBM-PC associated with a decrease of } 44 \% \\
\text { in } \\
\text { stunting, } 24 \% \text { in wasting, and } 26 \% \text { SGA } \\
\text { when compared to the control group; the } \\
\text { difference between LBM-FT and control } \\
\text { group was marginal }\end{array}$ & $\begin{array}{l}\text { Home visitor } \\
\text { research } \\
\text { assistants }\end{array}$ & $\begin{array}{l}\text { Increased } \\
\text { compliance } \\
\text { with } \\
\text { supplements } \\
\text { in the } \\
\text { intervention } \\
\text { arms (more in } \\
\text { the first arm } \\
\text { than the } \\
\text { second) }\end{array}$ \\
\hline
\end{tabular}

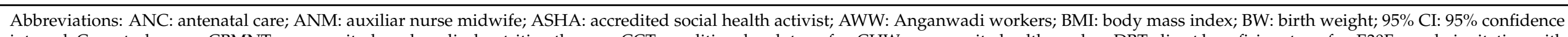

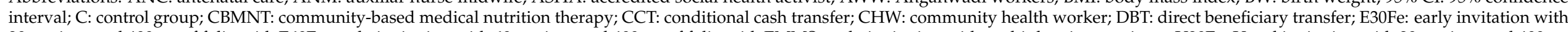

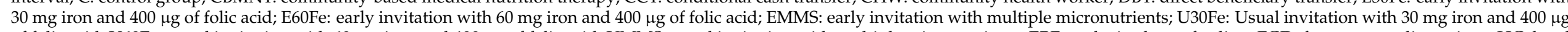

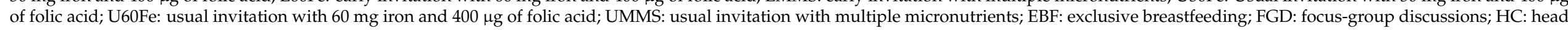

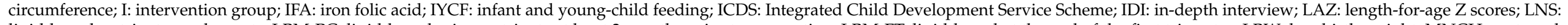

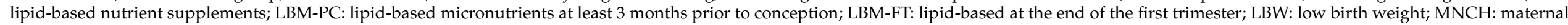

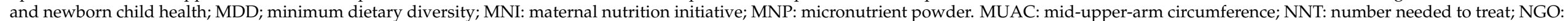

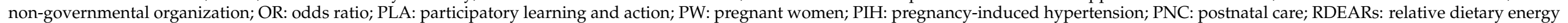

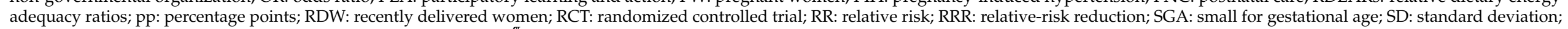

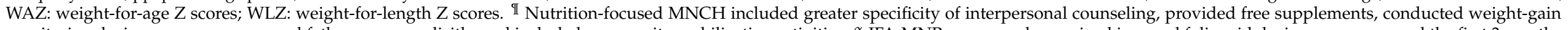

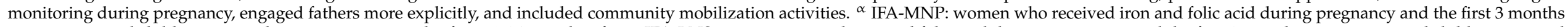

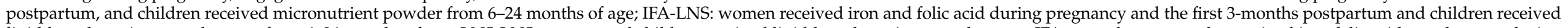

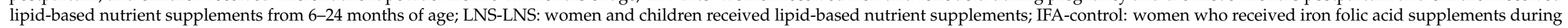

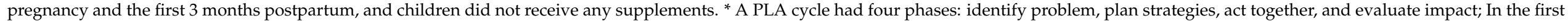

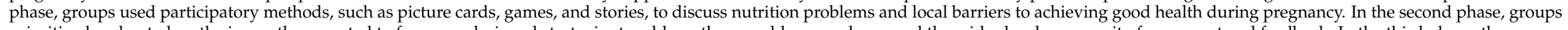

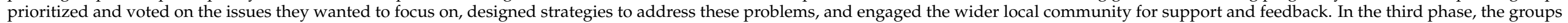

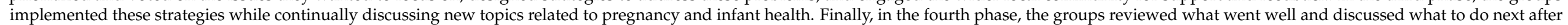

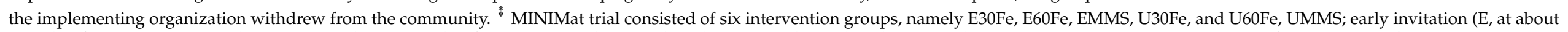

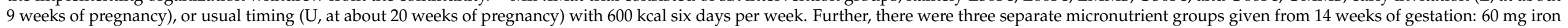

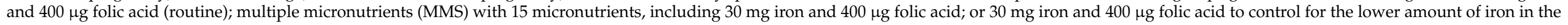
MMS supplement. 


\subsection{Summary of Interventions}

These studies include interventions of micronutrient supplementation (IFA, Calcium, and multiple micronutrients), deworming, nutrition education and counseling, BEP supplementation, and delivery mechanisms for implementing these interventions. The strategies adopted in these reviews were home visits, directly observed supplement intake, community mobilization, food and cash transfer, community volunteers, and peer-group education. Almost all interventions used community health workers as the primary human resource for delivering interventions to beneficiaries. Nearly two thirds of the studies showed improvement in the uptake/coverage/compliance with the intervention or services. Tables S6 and S7 (Supplementary File) give an overview of the findings for each article included in the narrative synthesis.

\section{a. Micronutrient supplementation (IFA, calcium, and multiple micronutrients)}

Community health workers, female health workers, research assistants, and community volunteers are crucial for delivery of nutrition supplements. Home visits, in particular, for delivering nutrition supplementation by various grassroots-level workers were also reported. IFA supplementation programs in Pakistan and Nepal were delivered by community health workers, and coverage of IFA supplements significantly increased in the last ten years, from $23 \%$ to $80 \%$ in Nepal. However, in Pakistan, coverage remained stagnant, at $45 \%$. Analysis also recorded IFA consumption with respect to the number of antenatal care (ANC) visits in Pakistan. IFA consumption of $45 \%$ was recorded among those who had at least one ANC visit, compared to $9.7 \%$ with no ANC visit; hence, ANC visit is a critical factor in this intervention [22].

IFA supplementation delivered by trained female health workers through a fortnightly home visit and a quarterly community-based group session reported that the proportion of intake of supplements was $>76 \%$, which was quite good, considering $65 \%$ had irondeficiency anemia and low-birth-weight prevalence ranged from $18 \%$ to $40 \%$ [17]. An evaluation of the intervention package delivered through the trained community health workers and local health workers for improving perinatal and neonatal outcomes through community mobilization, education and home visits, use of information education and communication materials, videos, community health committees, and group meetings reported improved ANC services, including IFA and calcium supplementation [25].

Specially trained community health workers were reported to be the frontline facilitators delivering maternal nutrition services at the household, community, or facility level. MANOSHI, a community-based maternal, neonatal, and child health care service package among slum dwellers in Bangladesh delivered through female health workers through monthly home visits for IFA, calcium supplementation, and nutrition education, reported an increase in IFA consumption by $19 \%(p<0.01)$ [28]. Similarly, the Government of Chhattisgarh, India, used community health workers, called mitanin (a local term for a close female friend), for improving the coverage of reproductive and child health services. Later, Nutrition Security Innovation, a project to inform families regarding their entitlement from the public distribution system, was integrated with this mitanin program to promote the appropriate complementary feeding through mitanins. An increased IFA consumption rate of 3\% was reported, with a low occurrence of side effects [23].

Trained community health workers were recruited for delivery of ANC services, including nutrition-related services in Bangladesh through home visits or at community centers; their actions resulted in increased IFA consumption, with more than $82 \%$ of participants consuming more than 90 calcium tablets for more than three months. The study also reported a significant difference in the consumption of calcium tablets in relation to number of ANC visits; women who had four or more ANC checkups consumed more calcium tablets $(p<0.001)$ [39].

Directly observed consumption of supplements by the beneficiaries in the presence of community health workers was also associated with an increased consumption rate of multiple micronutrients (68\%) and IFA (71\%), indicating that successful distribution of supplements and direct observation of consumption of supplements improved adherence 
to consumption [18]. Additionally, a weekly home visit by community health workers to deliver micronutrient supplements was found to be associated with high compliance with supplements (median: 95, interquartile Range: 89.1, 98.4) [24]. Maternal nutrition knowledge and support from husbands were the key maternal and household factors associated with higher consumption of IFA and calcium tablets. Health service factors, like early and more prenatal care visits, ANC, and receipt of free supplements, also improved the consumption rate; for every home visit conducted by female health workers, four additional IFA and five additional calcium tablets were consumed by mothers. Combined exposure to these factors-knowledge, family support, and self-efficacy-was attributed to consumption of an additional 46 IFA and 53 calcium tablets, with $68 \%$ of pregnant women achieving minimum dietary diversity. Thus, regular home visits for delivery of micronutrient supplements at the doorsteps ensured that every pregnant woman received adequate supplementation [32]. Secondary analysis of an intervention study from India and Pakistan, where lipid-based micronutrient was provided through research assistants, showed a significant improvement in length-for-age Z-score (LAZ), weight-for-age Z-score, and reduction in low birth weight, as well as small-for-gestational-age incidence [47].

\section{b. Nutrition education and counselling}

Nutrition education and counselling provided by frontline health workers were informative and useful when provided by the mitanins in Chhattisgarh's mitanin program. It was reported that a significant number of households among the intervention group adopted kitchen gardening ( $46.6 \%$ vs. $32.5 \%)$, and more than $98 \%$ reported having received information on the importance of IFA consumption. Involvement of community volunteers in family-level counselling for behavioral change and for delivery of nutrition services was also effective, and monthly nutrition and health education provided by Anganwadi workers (community-based frontline workers who promote child growth and development) during home visits or during village health and nutrition days was associated with $35 \%$ increased coverage in delivery of pregnancy care and nutrition information $[23,27,41]$. Studies from India and Bangladesh on health and nutrition programs showed that the trained community health workers were crucial in imparting nutrition education and counselling in these countries and were reported to have significantly improved the calcium-supplement consumption rate [20,39]. Monthly home visits by community health workers in Bangladesh to deliver nutrition education interventions resulted in 73.3\% of pregnant women reporting having had visits from community health workers, and nearly three-quarters of pregnant women could name at least five food groups that should be consumed daily. However, large knowledge-to-practice gaps were observed in terms of food consumption [32]. A similar study evaluated the effect of providing intensified nutrition-focused maternal, neonatal, and child health intervention compared to standard interventions. More than $96 \%$ of pregnant women in the intensified focused nutrition group received IFA and calcium tablets, and $40-50 \%$ of all mothers reported exposure to video shows. Proportion of IFA and calcium intake, number and quantity of food groups consumed, and daily intake of macronutrients improved substantially in the intensified focused nutrition group, as compared to the control group [33].

The participatory learning and action approach engages communities in learning and participation, identifying needs, planning, nutrition education, and counselling, and encouraging problem-solving. Studies were designed to assess the effects of pregnancyfocused nutrition interventions through the participatory learning and action approach, combined with the transfer of food or cash in intra-household food allocation, dietary adequacy, and maternal nutritional status in Nepal. Monthly group sessions were conducted in all the arms. The participatory approach used picture cards and stories for group discussions. A structured manual was prepared for nutrition mobilizers to conduct home visits and nutrition counselling to improve nutrition practice. The participatory learning and action approach with cash or food transfer reported a higher attendance rate at monthly meetings $(80 \%)$ compared to the approach alone without cash or food transfer. The participatory learning and action approach with food transfer significantly improved 
the birth weight of the fetus and equity in energy allocation among pregnant women. It also improved dietary diversity and adequacy, as well as supplement consumption [37,40]. Conditional cash transfer of INR 5000 (Indian Rupee) delivered in four installments (1500 at end of second trimester, 1500 at third month after delivery, 1000 at the sixth month, and 1000 at the ninth month) was reported from India. Each installment was conditional on the uptake of certain health care services and outcomes. Compliance with conditions, such as consumption of IFA tablets; counselling during pregnancy; and the recommended immunization for bacillus Calmette-Guerin, polio, and diphtheria-pertussis-tetanus were observed to be significantly higher (unadjusted $p<0.01$ ) among those receiving conditional cash transfer. Conditional cash transfer was also observed to be associated with an increased likelihood of pregnancy registration and receiving ANC services and IFA tablets from female health workers [34]. Counselling on maternal nutrition through home visits and participatory women's group meetings facilitated by community health workers at least twice a month was associated with a significant increase in the odds of pregnant women achieving minimum dietary diversity in intervention clusters (adjusted odds ratio $1.40 ; 95 \%$ CI 1.03 to $1.90, p=0.0311$ ) [36].

For the promotion of maternal, infant, and young-child nutrition, the components of behavior-change communication were integrated into agricultural extension programs on nutrition by developing cost-effective, video-based intervention. This showed that intake of IFA tablets was $86 \%$ among those who had received information, and several female health workers requested that the videos be disseminated during village health and nutrition days and during the monthly health and nutrition fairs. Female health workers also reported that the videos further helped in serving the community well. Interviews with various health workers, self-help groups, volunteers, and beneficiaries reported an increase in uptake and awareness of government services for improving nutrition [29].

\section{c. Balanced energy-protein supplementation}

Although evidence to evaluate potential long-term outcomes is not adequate, a few studies reported that supplementation of BEP diet in undernourished pregnant women facilitated gestational weight gain and improved fetal outcomes in terms of reducing the risk of stillbirth, low-birth-weight infants, and small-for-gestational-age babies. In Nepal, the provision of $10 \mathrm{~kg}$ per month of a fortified BEP supplement of wheat-soya blended flour with $10 \%$ added sugar, called super cereal, delivered by community health workers, along with the participatory learning and action approach, significantly improved the birth weight of newborns and equity in energy allocation among pregnant women [37,40]. Similarly, in Bangladesh, a locally produced food-based BEP supplementation for undernourished pregnant women delivered along with nutrition education and counselling and regularly monitored by community nutrition volunteers either at the designated center in the community or through home visits showed a $98 \%$ compliance rate, with almost all women consuming the full supplement daily [43]. However, evaluation of supplementary food programs delivered in India through the Integrated Child Development Service Scheme by Anganwadi workers reported that only $20.5 \%$ of women received supplementary food during their pregnancies due to a weak procurement system and poor quality of food products [20]. Another study from India and Pakistan provided additional protein-energy supplements to pregnant women whose BMI was $<20 \mathrm{~kg} / \mathrm{m}^{2}$ only in the interventional groups. LAZ, weight-for-age $\mathrm{z}$ score, low birth weight, and small-for-gestational-age were much improved in the interventional groups as compared to the control group. Prevalence of newborn stunting ( $\mathrm{LAZ}<-2)$ was $18 \%$ (Intervention 1: $10 \%$ \& Intervention 2: 13\%), wasting (weight-to-length ratio for age $<-2) 42 \%$ (32\% \& $37 \%)$, preterm deliveries $12 \%(12 \% \& 8.5 \%)$, low birth weight $34 \%(28 \% \& 29 \%)$ and small for gestational age $49 \%(36 \% \& 44 \%)$ in the control group, as compared to the intervention groups [47]. 


\section{d. Barriers affecting coverage, uptake, and compliance of program delivery}

Public health interventions are complex, and outcomes are unpredictable. Factors affecting the success of the programs are multidimensional. A few barriers to interventions were reported in some of the studies identified for this scoping review. An evaluation of the implementation of maternal nutrition programs in India reported that only $27.6 \%$ consumed at least 100 IFA tablets or syrup during their most recent pregnancy, only $4 \%$ took deworming medicine during pregnancy, $20.5 \%$ received supplementary food, and $10.9 \%$ received nutrition and health education. Similarly, the report recorded a meager utilization rate of the nutrition services provided at anganwadi centers and food-fortification projects implemented through the public distribution system. Inadequate and irregular supply of supplements, substandard quality of the food provided, misconceptions about intake of certain food items during pregnancy, and sharing of take-home rations with the household members were a few barriers that hindered program effectiveness [20]. Other factors that affected the delivery and uptake of interventions were resource shortages, socioenvironmental issues, including poverty, lack of awareness, and discrimination based on socioeconomic status in the community [21].

Certain policy-related barriers reported in different studies were low prioritization of maternal-nutrition intervention by policymakers and lack of proper thematic knowledge of the program reference. All these barriers were found to be associated with inefficient program management within the overall health system. One study also reported a low consumption rate of deworming pills $(4 \%)$, despite receiving the targeted intervention, due to weak monitoring, evaluation, and not prioritizing time-bound targets [21]. Barriers to IFA consumption were reported to be a lack of appropriate need forecasting, delay in supply, inconsistent training on IFA counselling/distribution, low health literacy, unplanned pregnancy, no or late pregnancy registration, limited intervention resources, and misconceptions [42,45].

\section{Discussion}

This review is different from other reviews conducted on maternal-nutrition intervention in several ways. The objective of this review was not to identify evidence-based maternal-nutrition interventions but to document intervention strategies that have successfully improved coverage and uptake of maternal nutrition services and programs. Review of implementation of various evidence-based interventions to improve maternal nutrition and birth outcomes indicates that community health workers, home visits, directly observed nutrition supplementation, community mobilization, and social marketing approaches for delivery of interventions like IFA supplementation, deworming, BEP supplementation, and nutrition education and counselling provide better results in terms of coverage, compliance with services and uptake of these interventions. The present review qualitatively narrates various intervention strategies adopted to deliver programs to improve maternal nutrition and birth outcomes.

\subsection{Effect of Home Visits by CHW on Compliance with Nutrition Interventions}

Maternal nutrition status is crucial for fetal growth and development; nutrition supplements are effective in improving maternal and fetal outcomes and have been routinely administered to pregnant women [48-50]. The review found that auxiliary nurse midwives, female health workers, community health workers, Anganwadi workers, accredited social health activists, and community volunteers all play a crucial role in delivering public health interventions either at a designated facility or community centers, on a designated day or through house visits. Reported outcomes of various studies showed an increase in supplement consumption rate when nutrition services were delivered by community health workers through home visits $[17,18,22,23]$. These findings support other reviews reporting on the delivery of services through home visits by community health workers. Thus, by ensuring universal health care with strengthened primary health services, the prevalence of anemia and undernutrition could be reduced [6]. Sanghvi et al. 2016 [51] 
examined evidence of IFA supplementation and the effectiveness of intervention trials in a large-scale program and found that the active involvement of village health volunteers through home visits helps to improve compliance and ANC visits.

Mason et al. 2012 [52] assessed the current scenario of IFA supplementation in India and reported that community health workers and auxiliary nurse midwives were the driving force for delivery of supplementation and observed an improvement in the uptake of services and intervention coverage. A recent population-based intervention study conducted by Edmond et al. (2018) [53] in Afghanistan evaluated the effectiveness of a program of home visits by trained community health workers and reported an increase in institutional delivery of $8.2 \%$ in the intervention group, as compared to $6.3 \%$ in the control group. There was also an increase in ANC visits of $3.4 \%$ in the intervention group, whereas a decline of $1 \%$ was observed in the control group. The findings show that home visits by community health workers improved care-seeking behavior and knowledge among pregnant women. Overall, home visits by community health workers increased coverage and compliance with the consumption of micronutrient supplements and uptake of ANC services.

Many studies lack data on the direct effect of health workers on the uptake of services/compliance (as revealed through association/regression/attribution) $[12,18,19,23$, $24,26,29,32-35,37,38,42,43]$. These studies compared the effect of the intervention (as a whole) on the outcomes without delineating the isolated effects of health workers/delivery mechanisms. However, some studies reported on the impact of CHW on health outcomes. In the MANOSHI program, $\mathrm{CHW} /$ trained providers/medically trained providers who paid monthly antenatal and postnatal visits reported greater effect of $\mathrm{CHW}$ on improving the uptake of four or more ANC visits and quality ANC visits than the routine services [28]. Frontline workers, like ASHA/AWW who were of the same caste as the head of the household and were living in the same catchment area of pregnant women, had higher odds of providing immunization services to children under 5 years. and pregnancy-related counselling, respectively [27]. CHWs trained on additional curriculum of newborn care and with improved counselling skills enhanced maternal and newborn care practices significantly compared to those who did not receive additional training and skill development [25]. However, CHW visits in addition to existing AWW were not found to significantly affect child health outcomes [36].

\subsection{Effects of Nutrition Education and Counselling on Compliance and Uptake of Nutrition Services or Intervention}

Existing evidence suggests that nutrition education and counselling is an effective strategy, but the impact is more significant and substantial among undernourished populations of low- and middle-income countries when accompanied by nutritional services, like provision of free micronutrient supplements, ANC care, periodic reinforcements, and food supplementation [54,55]. Nutrition education and counselling is the primary responsibility of community health workers and auxiliary nurse midwives, and every nutrition intervention and program has a component of nutrition education and counselling either at the individual, household, community or facility level. Delivery of nutrition education by trained community health workers or female health workers was reported to be associated with good nutrition knowledge, greater support from the husband, higher consumption of supplements, and achievement of minimum dietary diversity [20,27,32,39]. Home-based counselling, group meetings, and community events provided by frontline workers were also reported to be informative and useful [41]. A review by Girard et al. 2012 [51] reported that nutrition education and counselling targeting maternal diet and supplement intakes during pregnancy helped to improve dietary patterns and adherence to a healthy diet. Studies have also reported that nutrition education and counselling increased compliance with micronutrient supplementation and uptake and utilization of ANC services, which is in line with the findings of the present study. Another review [56] reported that large-scale programs involving nutrition education and counselling usually delivered by community health workers and a program in Bangladesh on nutritional counselling for behavior change succeeded in creating awareness and attention towards undernutrition 
and in designing a sustainable nutrition program in the country. Similarly, a review by Vaivada et al. [57] reported that nutrition education and counselling for pregnant women in undernourished populations was effective in improving birth outcomes and home visits by specially trained community health workers for counselling and IFA supplement and facilitates decision making concerning IFA consumption [58].

Participatory learning and action (PLA) in combination with cash or food transfer led to higher attendance rates at monthly meetings, improved birth weight, and equity in energy allocation among pregnant women, while PLA with cash transfer improved adequate dietary diversity and supplement consumption [37,40]. Community interventions through home visits and PLA by local community-based workers significantly achieved adequate dietary diversity among pregnant women [36,42]. In concurrence with this rapid review, a non-RCT by Gope et al. 2019 [59] weighed the effects of two strategies concerning monthly PLA meetings with women's groups followed by home visits and crèches combined with monthly PLA meetings and home visits. Trained community health workers facilitated the implementation, and the study reported that $65-72 \%$ of mothers participated in the meetings, and an increase in the uptake of services, early initiation of breastfeeding, adequate dietary diversity among mothers, and consumption of iron-rich foods by children were recorded. PLA meetings, along with home visits, reduced undernutrition among children, suggesting the potential benefit of scaling up the intervention through accredited social health activists and their facilitators.

\subsection{Effects of Food or Cash Transfer on Compliance and Uptake of Nutrition Services}

Take-home rations and supply of fortified food through the public distribution system was one of the interventions used to address maternal undernutrition and low birth weight, [21] but a study from India revealed that $35 \%$ of the sample households received food supplements from Anganwadi workers and accredited social health activists, and only $20.5 \%$ women reported to have received supplementary food during their pregnancy through the integrated child development service scheme (ICDS) program [20,27]. Inversely, in Bangladesh, the delivery of locally produced prenatal food-based supplements to pregnant women led to a high compliance rate, with almost all participants consuming the full supplement on a daily basis [43]. Similarly, in Nepal, food and cash transfer with PLA led a high participation rate in program activities and significant improvement in birth weight of newborns, equity in energy allocation, and adequate dietary diversity $[37,40]$. Earlier reviews found conditional cash transfer to be a better intervention strategy than direct distribution of food supplements. Mexico and Brazil succeeded in conditional cash transfer programs, and this approach is now being endorsed and implemented in other low- and middle-income countries [54,56].

Another study from Mexico [60] on the nutritional impact of a large-scale, incentivebased development program (PROGRESA), which includes the provision of micronutrient fortified food supplements to eligible women and cash transfers of USD 25 per family, with the condition of complying with specific healthcare appointments, including a mandatory session on nutrition and health education, showed an overall improvement in the consumption of nutrient supplements, improvement in height, and reduction in anemic cases. This indicates the potential benefit of the program for improving adherence to the intervention.

In India, the Janani Suraksha Yojana (JSY) program provided cash benefits to pregnant women from low-income households to promote institutional delivery, which led to an increase in the utilization of ANC and skilled delivery. This, in turn, led to a decline in perinatal mortality. Conversely, the provision of conditional monthly food rations and micronutrient-fortified individual rations for daily consumption improved the participation rate by more than $95 \%$ for monthly group sessions. High attendance and participation rates indicate that delivery of targeted nutrition intervention through conditional food ration facilitates program effectiveness in achieving its desired outcomes [61,62]. 


\subsection{Effect of Community-Level Events, Social Marketing Campaigns or Group Sessions on Compliance and Uptake of Nutrition Services}

Village health and nutrition day (VHND) in India, maternal and child health weeks in Nigeria, and enhanced outreach strategies or community health days in Ethiopia are examples of campaign-based approaches for delivery of nutrition interventions. VHND, a monthly event usually organized at Anganwadi centers, provides nutritional services, such as nutrition education and counselling and fortified take-home food rations to pregnant women, lactating mothers, and children. In Ethiopia and Nigeria, pregnant women and lactating mothers are screened to determine targeted beneficiaries for food supplementation through health days or health-week campaigns, as the majority of the women is reported to miss ANC visits [54]. Similarly, several reports in this review also discussed social campaigns, community events, or designated days for delivery of interventions. VHND is a beneficial platform for reaching those who did not receive services through other platforms, utilization of information, education and communication materials for conducting community group meetings, home-based sessions for delivery of nutrition education messages, delivery of supplements and problem-solving through peer group discussions, etc. [20,25,42].

Community-level campaigns were found to be effective in creating awareness and promoting the intake of supplements. Social marketing and community-based education sessions using information, education, and communication material before distribution of supplements were found to be effective in the uptake and usage of supplements [19]. Similar interventions reported that the involvement of community health workers in community mobilization activities and home visits helps in tracking uptake and utilization of nutritional services and identifying defaulters [63]. A study from Africa reported an increase in the utilization rate of healthcare-related services following intensive and exhaustive community-mobilization and advocacy activities [64]. Similarly, a study from Vietnam promoting IFA consumption reported community mobilization and social marketing as an effective intervention strategy for promotion of uptake of services and intervention coverage. The study also reported a significant change in the knowledge, attitude, and practice of the targeted beneficiaries related to health and nutrition [65].

\subsection{Strengths and Limitations}

We have identified a wider range of maternal nutrition programs and their implementation through a stringent process to include them in this review. However, only PubMed and Google Scholar were searched to identify articles, as there was a lack of time and resources. Owing to this, there could be a greater chance of missing out on other relevant studies not indexed in PubMed or Google Scholar. Additionally, meta-analysis was not performed in the present paper due to varied outcome indicators and non-uniformity in the presentation of results across studies. However, the studies included in the review present a diverse range of strategies to improve maternal nutrition in many countries, including India, Pakistan, Indonesia, etc., compared to the previous reviews. Furthermore, many aspects of maternal nutrition programs were discussed, including strategies, effectiveness to improve maternal and child health outcomes, and delivery mechanisms. This makes this paper relevant for policy makers, public health specialists, and academicians.

\section{Conclusions}

Specific interventions targeting maternal nutrition have proven to be effective in improving pregnancy outcomes. However, the proportion of people who benefitted from these interventions in South-East Asian countries, with a high prevalence of anemia and underweight women and low rates of IFA consumption, [66] needs to be explored. Implementing public health programs, small or large, is complex and requires an understanding of cultural diversity, the public health system, and population characteristics, and program implementers should examine the benefits of effective intervention strategies for context-specific and effective program design. 
In this review, intervention strategies, such as home visits, nutrition education, and counselling through different means, including video-based sessions, free supplementation of IFA/calcium tablets, monitoring of ANC visits, and community mobilization and meetings, were found to be associated with higher intervention coverage, increased compliance with intervention, and uptake of services. However, intervention strategies such as food rations and food transfer showed varied effects. Studies from India reported that the quality of food supplied under food-transfer programs was not suitable for consumption, which inversely affected program coverage. Cash transfer, on the other hand, led to improved compliance with interventions, as reported by studies from Nepal, although evidence was not definitive and needs further evaluation.

The overall findings from this scoping review were that strengthening of ANC at the community or facility level to promote ANC visits, frequent home visits, building capacity of community health workers, promoting participatory community mobilization, nutrition campaigns, conditional cash transfer, provision of food supplements, and improving quality of food supplements improve coverage, compliance, and uptake of services. Furthermore, there is a need to improve quality and regular supply and address the shortage of food supplements, with continuous monitoring. Solutions to address barriers related to program delivery are needed to further improve effectiveness. Besides, the socioenvironmental factors such as poverty, lack of awareness, and misconceptions in the community require immediate action. The findings of this review will help decision makers and program implementers who seek to understand the complexity of implementing such evidencebased interventions addressing maternal undernutrition and birth outcomes for effective program design and improvement of program efficiency and effectiveness.

Supplementary Materials: The following are available online at https:/ / www.mdpi.com/article/ 10.3390/ijerph182413292/s1, Figure S1. Nutrition search strategy in PubMed; Table S1. Quality Appraisal Checklist for Randomized Controlled Trials; Table S2. Quality Appraisal Checklist for Analytical Cross-Sectional Studies; Table S3. Quality Appraisal Checklist for Quasi-Experimental Studies; Table S4. Quality Appraisal Checklist for Qualitative Research; Table S5. Quality Appraisal Checklist for Cohort Studies; Table S6. Characteristics of the included studies; Table S7. Summary of findings.

Author Contributions: T.L., R.K.S. and S.M. conceptualized the scoping review; T.L., K.K., R.K.S., N.K., P.K. and S.M. designed the study; K.K., T.L., P.K., N.K. and R.K.S. identified, selected, and extracted data from the included articles; K.K., T.L. and R.K.S. appraised the quality of the studies; K.K. and T.L. wrote the first draft of the manuscript; S.M., S.S. and D.M. gave critical and overall comments about the manuscript; S.M., S.S., D.M. and K.K. drafted the final version of the manuscript. All authors have read and agreed to the published version of the manuscript.

Funding: This research received no external funding.

Institutional Review Board Statement: Not applicable.

Conflicts of Interest: The authors declare no conflict of interest.

\section{References}

1. Ahmed, T.; Hossain, M.; Sanin, K.I. Global Burden of Maternal and Child Undernutrition and Micronutrient Deficiencies. Ann. Nutr. Metab. 2012, 61 (Suppl. S1), 8-17. [CrossRef]

2. Christian, P.; Smith, E.R.; Zaidi, A. Addressing inequities in the global burden of maternal undernutrition: The role of target-ing. BMJ Glob. Health 2020, 5, e002186. [CrossRef]

3. Development Initiatives. Global Nutrition Report 2017: Nourishing the SDGs; Development Initiatives: Bristol, UK, 2017.

4. Directorate of Community Nutrition, Ministry of Health. Atmarita: Nutrition problems in Indonesia. In Proceedings of the Integrated International Seminar and Workshop on Lifestyle-Related Diseases 2005, Yogyakarta, Indonesia, $19-20$ March 2005.

5. Swaminathan, S.; Hemalatha, R.; Pandey, A.; Kassebaum, N.J.; Laxmaiah, A.; Longvah, T.; Lodha, R.; Ramji, S.; Kumar, G.A.; Afshin, A.; et al. The burden of child and maternal malnutrition and trends in its indicators in the states of India: The Global Burden of Disease Study 1990-2017. Lancet Child Adolesc. Health 2019, 3, 855-870. [CrossRef]

6. Bellizzi, S.; Pichierri, G.; Napodano, C.M.P.; Salaris, P.; Fiamma, M.; Fozza, C.; Cegolon, L. Iron deficiency anaemia and low BMI among adolescent girls in India: The transition from 2005 to 2015. Public Health Nutr. 2021, 24, 1577-1582. [CrossRef] 
7. Black, R.E.; Allen, L.H.; Bhutta, Z.A.; Caulfield, L.E.; De Onis, M.; Ezzati, M.; Mathers, C.; Rivera, J.; Maternal and Child Undernutrition Study Group. Maternal and child undernutrition: Global and regional exposures and health consequences. Lancet 2008, 371, 243-260. [CrossRef]

8. Kumera, G.; Gedle, D.; Alebel, A.; Feyera, F.; Eshetie, S. Undernutrition and its association with socio-demographic, anemia and intestinal parasitic infection among pregnant women attending antenatal care at the University of Gondar Hospital, Northwest Ethiopia. Matern. Health Neonatol. Perinatol. 2018, 4, 18. [CrossRef] [PubMed]

9. Dadi, A.F.; Desyibelew, H.D. Undernutrition and its associated factors among pregnant mothers in Gondar town, Northwest Ethiopia. PLoS ONE 2019, 14, e0215305. [CrossRef] [PubMed]

10. Endalifer, M.; Tewabe, M.; Adar, A. Undernutrition and associated factors among pregnant women attending ANC follow up in Alamata general hospital, Northern Region, Ethiopia, 2017. J. Nutr. Health Food Eng. 2019, 9, 70-78.

11. Bhutta, Z.A.; Gupta, I.; De'Silva, H.; Manandhar, D.; Awasthi, S.; Hossain, S.M.M.; Salam, M.A. Maternal and child health: Is South Asia ready for change? BMJ 2004, 328, 816-819. [CrossRef] [PubMed]

12. Perez-Escamilla, R.; Bermudez, O.; Buccini, G.S.; Kumanyika, S.; Lutter, C.K.; Monsivais, P.; Victora, C. Nutrition disparities and the global burden of malnutrition. BMJ 2018, 361, k2252. [CrossRef]

13. Goudet, S.; Murira, Z.; Torlesse, H.; Hatchard, J.; Busch-Hallen, J. Effectiveness of programme approaches to improve the cover-age of maternal nutrition interventions in South Asia. Matern. Child Nutr. 2018, 14 (Suppl. S4), e12699. [CrossRef] [PubMed]

14. Blencowe, H.; Krasevec, J.; de Onis, M.; Black, R.E.; An, X.; Stevens, G.A.; Borghi, E.; Hayashi, C.; Estevez, D.; Cegolon, L.; et al. National, regional, and worldwide estimates of low birthweight in 2015, with trends from 2000: A systematic analysis. Lancet Glob. Health 2019, 7, e849-e860. [CrossRef]

15. Tricco, A.C.; Lillie, E.; Zarin, W.; O’Brien, K.K.; Colquhoun, H.; Levac, D.; Moher, D.; Peters, M.D.J.; Horsley, T.; Weeks, L.; et al. PRISMA extension for scoping reviews (PRIS-MA-ScR): Checklist and explanation. Ann. Intern. Med. 2018, 169, 467-473. [CrossRef] [PubMed]

16. Joanna Briggs Institute. Critical Appraisal Tools. Available online: https://joannabriggs.org/critical-appraisal-tools (accessed on 15 November 2019).

17. Bhutta, Z.A.; Rizvi, A.; Raza, F.; Hotwani, S.; Zaidi, S.; Hossain, S.M.; Soofi, S.; Bhutta, S. A Comparative Evaluation of Multiple Micronutrient and Iron-Folic Acid Supplementation during Pregnancy in Pakistan: Impact on Pregnancy Outcomes. Food Nutr. Bull. 2009, 30, S496-S505. [CrossRef] [PubMed]

18. Wang, S.; Utomo, B.; Hidayat, A.; Subarkah, K. Preventing low birthweight through maternal multiple micro-nutrient supplementation: A cluster-randomized, controlled trial in Indramayu, West Java. Food Nutr. Bull. 2009, 30 (Suppl. S4), S488-S495. [CrossRef]

19. Rah, J.H.; de Pee, S.; Halati, S.; Parveen, M.; Mehjabeen, S.S.; Steiger, G.; Bloem, M.W.; Kraemer, K. Provision of Micronutrient Powder in Response to the Cyclone Sidr Emergency in Bangladesh: Cross-Sectional Assessment at the end of the Intervention. Food Nutr. Bull. 2011, 32, 277-285. [CrossRef]

20. Ramakrishnan, U.; Lowe, A.; Vir, S.; Kumar, S.; Mohanraj, R.; Chaturvedi, A.; Noznesky, E.A.; Martorell, R.; Mason, J.B. Public health interventions, barriers, and opportunities for improving maternal nutrition in India. Food Nutr. Bull. 2012, 33 (Suppl. S1), S71-S92. [CrossRef]

21. Noznesky, E.A.; Ramakrishnan, U.; Martorell, R. A Situation Analysis of Public Health Interventions, Barriers, and Opportunities for Improving Maternal Nutrition in Bihar, India. Food Nutr. Bull. 2012, 33, S93-S103. [CrossRef]

22. Nisar, Y.B.; Dibley, M.J. Earlier initiation and use of a greater number of iron-folic acid supplements during pregnancy pre-vents early neonatal deaths in Nepal and Pakistan. PLoS ONE 2014, 9, e112446. [CrossRef] [PubMed]

23. Vir, S.C.; Kalita, A.; Mondal, S.; Malik, R. Impact of community-based mitanin programme on undernutrition in rural Chhattisgarh State, India. Food Nutr. Bull. 2014, 35, 83-91. [CrossRef] [PubMed]

24. Gernand, A.D.; Schulze, K.J.; Nanayakkara-Bind, A.; Arguello, M.; Shamim, A.A.; Ali, H.; Wu, L.; West, K.P., Jr.; Christian, P. Effects of prenatal multiple micronutrient supplementation on fetal growth factors: A cluster-randomized, controlled trial in rural Bangladesh. PLoS ONE 2015, 10, e0137269. [CrossRef]

25. Memon, Z.A.; Khan, G.N.; Soofi, S.B.; Baig, I.Y.; Bhutta, Z.A. Impact of a community-based perinatal and newborn preventive care package on perinatal and neonatal mortality in a remote mountainous district in Northern Pakistan. BMC Pregnancy Childbirth 2015, 15, 106. [CrossRef] [PubMed]

26. Sablok, A.; Batra, A.; Thariani, K.; Batra, A.; Bharti, R.; Aggarwal, A.R.; Kabi, B.; Chellani, H. Supplementation of vitamin D in pregnancy and its correlation with feto-maternal outcome. Clin. Endocrinol. 2015, 83, 536-541. [CrossRef] [PubMed]

27. Kosec, K.; Avula, R.; Holtemeyer, B.; Tyagi, P.; Hausladen, S.; Menon, P. Predictors of essential health and nutrition service delivery in Bihar, India: Results from household and frontline worker surveys. Glob. Health Sci. Pract. 2015, 3, 255-273. [CrossRef] [PubMed]

28. Jolly, S.P.; Rahman, M.; Afsana, K.; Yunus, F.M.; Chowdhury, A.M.R. Evaluation of Maternal Health Service Indicators in Urban Slum of Bangladesh. PLoS ONE 2016, 11, e0162825. [CrossRef]

29. Kadiyala, S.; Morgan, E.H.; Cyriac, S.; Margolies, A.; Roopnaraine, T. Adapting Agriculture Platforms for Nutrition: A Case Study of a Participatory, Video-Based Agricultural Extension Platform in India. PLoS ONE 2016, 11, e0164002. [CrossRef] 
30. Mridha, M.K.; Matias, S.L.; Chaparro, C.M.; Paul, R.R.; Hussain, S.; Vosti, S.A.; Harding, K.L.; Cummins, J.R.; Day, L.T.; Saha, S.L.; et al. Lipid-based nutrient supplements for pregnant women reduce newborn stunting in a cluster-randomized controlled effectiveness trial in Bangladesh. Am. J. Clin. Nutr. 2016, 103, 236-249. [CrossRef]

31. Rahman, M.; Yunus, F.M.; Shah, R.; Jhohura, F.T.; Mistry, S.K.; Quayyum, T.; Aktar, B.; Afsana, K. A Controlled Before-and-After Perspective on the Improving Maternal, Neonatal, and Child Survival Program in Rural Bangladesh: An Impact Analysis. PLoS ONE 2016, 11, e0161647. [CrossRef]

32. Nguyen, P.H.; Sanghvi, T.; Kim, S.S.; Tran, L.M.; Afsana, K.; Mahmud, Z.; Aktar, B.; Menon, P. Factors influencing maternal nutrition practices in a large scale maternal, newborn and child health program in Bangladesh. PLoS ONE 2017, 12, e0179873. [CrossRef] [PubMed]

33. Nguyen, P.H.; Kim, S.S.; Sanghvi, T.; Mahmud, Z.; Tran, L.M.; Shabnam, S.; Aktar, B.; Haque, R.; Afsana, K.; Frongillo, E.A.; et al. Integrating Nutrition Interventions into an Existing Maternal, Neonatal, and Child Health Program Increased Maternal Dietary Diversity, Micronutrient Intake, and Exclusive Breastfeeding Practices in Bangladesh: Results of a Cluster-Randomized Program Evaluation. J. Nutr. 2017, 147, 2326-2337. [CrossRef]

34. Raghunathan, K.; Chakrabarti, S.; Avula, R.; Kim, S.S. Can conditional cash transfers improve the uptake of nutrition interventions and household food security? Evidence from Odisha's Mamata scheme. PLoS ONE 2017, 12, e0188952. [CrossRef] [PubMed]

35. Dewey, K.G.; Mridha, M.K.; Matias, S.L.; Arnold, C.D.; Cummins, J.R.; Khan, S.A.; Maalouf-Manasseh, Z.; Siddiqui, Z.; Ullah, B.; Vosti, S.A. Lipid-based nutrient supplementation in the first $1000 \mathrm{~d}$ improves child growth in Bangladesh: A cluster-randomized effectiveness trial. Am. J. Clin. Nutr. 2017, 105, 944-957. [CrossRef]

36. Nair, N.; Tripathy, P.; Sachdev, H.S.; Pradhan, H.; Bhattacharyya, S.; Gope, R.; Gagrai, S.; Rath, S.; Rath, S.; Sinha, R.; et al. Effect of participatory women's groups and counselling through home visits on children's linear growth in rural eastern India (CARING trial): A cluster-randomised controlled trial. Lancet Glob. Health 2017, 5, e1004-e1016. [CrossRef]

37. Harris-Fry, H.; Paudel, P.; Harrisson, T.; Shrestha, N.; Jha, S.; Beard, B.J.; Copas, A.; Shrestha, B.P.; Manandhar, D.S.; Costello, A.M.D.L.; et al. Participatory Women's Groups with Cash Transfers Can Increase Dietary Diversity and Micronutrient Adequacy during Pregnancy, whereas Women's Groups with Food Transfers Can Increase Equity in Intrahousehold Energy Allocation. J. Nutr. 2018, 148, 1472-1483. [CrossRef] [PubMed]

38. Hashmi, A.H.; Paw, M.K.; Nosten, S.; Darakamon, M.C.; Gilder, M.E.; Charunwatthana, P.; Carrara, V.I.; Wickramasinghe, K.; Angkurawaranon, C.; Plugge, E.; et al. 'Because the baby asks for it': A mixed-methods study on local perceptions toward nutrition during pregnancy among marginalised migrant women along the Myanmar-Thailand border. Glob. Health Action 2018, 11, 1473104. [CrossRef] [PubMed]

39. Khanam, F.; Hossain, B.; Mistry, S.K.; Mitra, D.K.; Raza, W.A.; Rifat, M.; Afsana, K.; Rahman, M. The association between daily $500 \mathrm{mg}$ calcium supplementation and lower pregnancy-induced hypertension risk in Bangladesh. BMC Pregnancy Childbirth 2018, 18, 406. [CrossRef]

40. Saville, N.M.; Shrestha, B.P.; Style, S.; Harris-Fry, H.; Beard, B.J.; Sen, A.; Jha, S.; Rai, A.; Paudel, V.; Sah, R. Impact on birth weight and child growth of Participatory Learning and Action women's groups with and without transfers of food or cash during pregnancy: Findings of the low birth weight South Asia cluster-randomised controlled trial (LBWSAT) in Nepal. PLoS ONE 2018, 13, e0194064. [CrossRef]

41. More, N.S.; Waingankar, A.; Ramani, S.; Chanani, S.; D’Souza, V.; Pantvaidya, S.; Fernandez, A.; Jayaraman, A. Community-Based Management of Acute Malnutrition to Reduce Wasting in Urban Informal Settlements of Mumbai, India: A Mixed-Methods Evaluation. Glob. Health Sci. Pract. 2018, 6, 103-127. [CrossRef]

42. Stevens, A.; Gilder, M.; Moo, P.; Hashmi, A.; Toe, S.; Doh, B.; Nosten, S.; Chotivanich, K.; Somerset, S.; Mcgready, R. Folate supplementation to prevent birth abnormalities: Evaluating a community-based participatory action plan for refugees and migrant workers on the Thailand-Myanmar border. Public Health 2018, 161, 83-89. [CrossRef]

43. Stevens, B.; Watt, K.; Brimbecombe, J.; Clough, A.; Judd, J.A.; Lindsay, D. A village-matched evaluation of providing a local supplemental food during pregnancy in rural Bangladesh: A preliminary study. BMC Pregnancy Childbirth 2018, 18, 286. [CrossRef]

44. Svefors, P.; Selling, K.E.; Shaheen, R.; Khan, A.I.; Persson, L.Å.; Lindholm, L. Cost-effectiveness of prenatal food and micronutrient interventions on under-five mortality and stunting: Analysis of data from the MINIMat randomized trial, Bangladesh. PLoS ONE 2018, 13, e0191260. [CrossRef]

45. Wendt, A.S.; Stephenson, R.; Young, M.F.; Verma, P.; Srikantiah, S.; Webb-Girard, A.; Hogue, C.J.; Ramakrishnan, U.; Martorell, R. Identifying bottlenecks in the iron and folic acid supply chain in Bihar, India: A mixed-methods study. BMC Health Serv. Res. 2018, 18, 281. [CrossRef] [PubMed]

46. Pavithra, G.; Kumar, S.G.; Roy, G. Effectiveness of a community-based intervention on nutrition education of mothers of mal-nourished children in a rural coastal area of South India. Indian J. Public Health 2019, 63, 4.

47. Dhaded, S.M.; Hambidge, K.M.; Ali, S.A.; Somannavar, M.; Saleem, S.; Pasha, O.; Khan, U.; Herekar, V.; Vernekar, S.; Kumar, Y.S. Preconception nutrition intervention improved birth length and reduced stunting and wasting in newborns in South Asia: The Women First Randomized Controlled Trial. PLoS ONE 2020, 15, e0218960. [CrossRef] [PubMed]

48. WHO: E-Library of Evidence for Nutrition Actions (eLENA). Balanced Energy and Protein Supplementation during Pregnancy. 2019. Available online: http://www.who.int/elena/titles/energy_protein_pregnancy/en/ (accessed on 22 February 2020). 
49. WHO: E-Library of Evidence for Nutrition Actions (eLENA). Daily Iron and Folic Acid Supplementation during Pregnancy. 2019. Available online: http:/ /www.who.int/elena/titles/daily_iron_pregnancy/en/ (accessed on 22 February 2020).

50. Kumar, A.; Kaur, S. Calcium: A Nutrient in Pregnancy. J. Obstet. Gynecol. India 2017, 67, 313-318. [CrossRef]

51. Sanghvi, T.G.; Harvey, P.W.; Wainwright, E. Maternal iron-folic acid supplementation programs: Evidence of impact and implementation. Food Nutr. Bull. 2010, 31 (Suppl. S2), S100-S107. [CrossRef]

52. Mason, J.B.; Saldanha, L.S.; Ramakrishnan, U.; Lowe, A.; Noznesky, E.A.; Girard, A.W.; McFarland, D.A.; Martorell, R. Opportunities for Improving Maternal Nutrition and Birth Outcomes: Synthesis of Country Experiences. Food Nutr. Bull. 2012, 33, S104-S137. [CrossRef]

53. Edmond, K.M.; Yousufi, K.; Anwari, Z.; Sadat, S.M.; Staniczai, S.M.; Higgins-Steele, A.; Bellows, A.L.; Smith, E.R. Can community health worker home visiting improve care-seeking and maternal and newborn care practices in fragile states such as Afghanistan? A population-based intervention study. BMC Med. 2018, 16, 106. [CrossRef]

54. Girard, A.W.; Olude, O. Nutrition Education and Counselling Provided during Pregnancy: Effects on Maternal, Neonatal and Child Health Outcomes. Paediatr. Périnat. Epidemiol. 2012, 26, 191-204. [CrossRef]

55. Garg, A.; Kashyap, S. Effect of counseling on nutritional status during pregnancy. Indian J. Pediatr. 2006, 73, 687-692. [CrossRef] [PubMed]

56. Victora, C.G.; Barros, F.C.; Assunção, M.C.; Restrepo-Méndez, M.C.; Matijasevich, A.; Martorell, R. Scaling up maternal nutrition programs to improve birth outcomes: A review of implementation issues. Food Nutr. Bull. 2012, 33 (Suppl. S1), S6-S26. [CrossRef]

57. Vaivada, T.; Gaffey, M.F.; Das, J.K.; Bhutta, Z.A. Evidence-based interventions for improvement of maternal and child nutrition in low-income settings: What's new? Curr. Opin Clin. Nutr. Metab. Care 2017, 20, 204-210. [CrossRef]

58. Alam, A.; Rasheed, S.; Khan, N.U.; Sharmin, T.; Huda, T.M.; Arifeen, S.E.; Dibley, M.J. How can formative research inform the design of an iron-folic acid supplementation intervention starting in first trimester of pregnancy in Bangladesh? BMC Public Health 2015, 15, 374. [CrossRef]

59. Gope, R.K.; Tripathy, P.; Prasad, V.; Pradhan, H.; Sinha, R.K.; Panda, R.; Chowdhury, J.; Murugan, G.; Roy, S.; De, M.; et al. Effects of participatory learning and action with women's groups, counselling through home visits and crèches on undernutrition among children under three years in eastern India: A quasi-experimental study. BMC Public Health 2019, 19, 962. [CrossRef]

60. Rivera, J.A.; Sotres-Alvarez, D.; Habicht, J.-P.; Shamah, T.; Villalpando, S. Impact of the Mexican program for education, health, and nutrition (Progresa) on rates of growth and anemia in infants and young children: A randomized effectiveness study. JAMA 2004, 291, 2563-2570. [CrossRef] [PubMed]

61. Olney, D.K.; Leroy, J.L.; Bliznashka, L.; Ruel, M. PROCOMIDA, a Food-Assisted Maternal and Child Health and Nutrition Program, Reduces Child Stunting in Guatemala: A Cluster-Randomized Controlled Intervention Trial. J. Nutr. 2018, 148, 1493-1505. [CrossRef]

62. Leroy, J.L.; Olney, D.; Ruel, M. Tubaramure, a Food-Assisted Integrated Health and Nutrition Program, Reduces Child Stunting in Burundi: A Cluster-Randomized Controlled Intervention Trial. J. Nutr. 2018, 148, 445-452. [CrossRef] [PubMed]

63. Olaniran, A.; Madaj, B.; Bar-Zev, S.; van den Broek, N. The roles of community health workers who provide maternal and new-born health services: Case studies from Africa and Asia. BMJ Glob. Health 2019, 4, e001388. [CrossRef]

64. Adah, S.; Ogbonna, C.; Anga, P.; Chingle, M.; Ashikeni, M.; Envuladu, E.; Agaba, C.; Audu, S.; Bupwatda, P.; Zoakah, A.J. The impact of advocacy and community mobilization on the utilization of health services at the Comprehensive Health Centre, Gindiri. Jos J. Med. 2009, 4, 11-13. [CrossRef]

65. Khan, N.C.; Thanh, H.T.K.; Berger, J.; Hoa, P.T.; Quang, N.D.; Smitasiri, S.; Cavalli-Sforza, T. Community mobilization and social marketing to promote weekly iron-folic acid supplementation: A new approach toward controlling anemia among women of reproductive age in Vietnam. Nutr. Rev. 2005, 63 (Suppl. S2), S87-S94. [CrossRef]

66. Chaparro, C.; Oot, L.; Sethuraman, K. India Nutrition Profile. 2014. Available online: https://www.fantaproject.org/sites/ default/files/download/India-Nutrition-Profile-Mar2014.pdf (accessed on 23 February 2020). 